Supplement of Biogeosciences Discuss., 12, 3283-3314, 2015

http://www.biogeosciences-discuss.net/12/3283/2015/

doi:10.5194/bgd-12-3283-2015-supplement

(C) Author(s) 2015. CC Attribution 3.0 License.

(c) (i)

Supplement of

\title{
Redox regime shifts in microbially-mediated biogeochemical cycles
}

T. Bush et al.

Correspondence to: T. Bush (t.bush@sms.ed.ac.uk) 


\section{Contents}

S1. Steady-state solutions for the fully biotic and biotic-abiotic nutrient-cycling models 2

Fully biotic model

Biotic-abiotic model

Abiotic-abiotic cycles

S2. Predictions for the microbial population density

S3. The role of the maximal population density parameter $n_{\max }$

Fully biotic cycle

Biotic-abiotic cycle

S4. Modelling explicit growth limitation by an external nutrient source

S5. Spatial heterogeneity accentuates redox regime shifts

S6. Redox regime shifts still occur with chemical sources and sinks

S7. Connection between our model and the Goldbeter-Koshland model for enzymatic phosphorylation-dephosphorylation cycles

S8. The four-population two-box model: explicit generation of acetate and oxygen

Equations and parameters for the fully biotic four-population, two-box model

Equivalent results for a biotic-abiotic model

S9. Redox regime shifts are preserved when intermediate chemical oxidation states are included

S10. Competition for oxygen and acetate 


\section{S1. STEADY-STATE SOLUTIONS FOR THE FULLY BIOTIC AND BIOTIC-ABIOTIC NUTRIENT-CYCLING MODELS}

In Eqs. (1-3) of the main text, we present dynamical equations for the model of Fig. 1e (blue arrows), for the case where both the reductive and oxidative reactions are mediated by microbial metabolism. We refer to this as the "fully biotic" model. Eqs. (1-2) and (6) describe an alternative model where the reductive step is microbe-mediated but the oxidative step is abiotic (Fig. 1e, red and blue arrows). We refer to this as the "biotic-abiotic" model. Here we present analytical results for the steady states of both of these models. These are the solutions that are plotted in Fig. 2 of the main text, except where the parameters are such that the analytical solutions predict negative values of the microbial population density; this is typically the case where the microbial maximal growth rate is smaller than the death rate. For these parameter combinations, the analytical solutions are not valid and instead the microbial population density should be set to zero.

\section{Fully biotic model}

The dynamical equations for the fully biotic model are

$$
\begin{gathered}
n_{\mathrm{or}}^{\cdot}=n_{\mathrm{or}}\left(\frac{v_{\mathrm{or}} s_{\mathrm{o}}}{s_{\mathrm{o}}+K_{\mathrm{or}}}\right)\left(1-\frac{n_{\mathrm{or}}}{n_{\mathrm{or}, \mathrm{max}}}\right)-d n_{\mathrm{or}} \\
n_{\mathrm{ro}}^{\cdot}=n_{\mathrm{ro}}\left(\frac{v_{\mathrm{ro}} s_{\mathrm{r}}}{s_{\mathrm{r}}+K_{\mathrm{ro}}}\right)\left(1-\frac{n_{\mathrm{ro}}}{n_{\mathrm{ro}, \mathrm{max}}}\right)-d n_{\mathrm{ro}} \\
\dot{s_{\mathrm{o}}}=\gamma n_{\mathrm{ro}}\left(\frac{v_{\mathrm{ro}} s_{\mathrm{r}}}{s_{\mathrm{r}}+K_{\mathrm{ro}}}\right)\left(1-\frac{n_{\mathrm{ro}}}{n_{\mathrm{ro}, \max }}\right)-\gamma n_{\mathrm{or}}\left(\frac{v_{\mathrm{or}} s_{\mathrm{o}}}{s_{\mathrm{o}}+K_{\mathrm{or}}}\right)\left(1-\frac{n_{\mathrm{or}}}{n_{\mathrm{or}, \max }}\right) \\
\dot{s_{\mathrm{r}}}=-\dot{s_{\mathrm{o}}}
\end{gathered}
$$

These equations correspond to Eqs. (1-3) of the main text. Here, $n_{\mathrm{or}}$ and $n_{\mathrm{ro}}$ are the population densities of the reducer and oxidizer populations respectively, $s_{\mathrm{O}}$ and $s_{\mathrm{r}}$ are the concentrations of the oxidized and reduced forms of the nutrient being cycled, $K_{\text {or }}$ and $v_{\text {or }}$ are the half-maximal nutrient concentration and maximal growth rate for the reducer population, $K_{\text {ro }}$ and $v_{\text {ro }}$ are the half-maximal nutrient concentration and maximal growth rate for the oxidizer population, $n_{\mathrm{or}, \max }$ and $n_{\text {ro,max }}$ are the respective maximal population densities, $d$ is the microbial death rate and $\gamma$ is the number of substrate concentration units required to form one biomass unit (i.e. a yield parameter). The latter two parameters are here assumed to be equal for the two populations, but similar phenomena are obtained if they are allowed to be different.

Setting $n_{\text {or }}$ and $n_{\text {ro }}$ to zero, we obtain

$$
d=\left(\frac{v_{\mathrm{or}} s_{\mathrm{o}}}{s_{\mathrm{o}}+K_{\mathrm{or}}}\right)\left(1-\frac{n_{\mathrm{or}}}{n_{\mathrm{or}, \max }}\right)=\left(\frac{v_{\mathrm{ro}} s_{\mathrm{r}}}{s_{\mathrm{r}}+K_{\mathrm{ro}}}\right)\left(1-\frac{n_{\mathrm{ro}}}{n_{\mathrm{ro}, \max }}\right),
$$

which, upon substitution into Eq. (S3), implies that in the steady state $n_{\text {or }}=n_{\text {ro }}$, i.e. the two population densities are equal.

Rearranging Eq. (S5) we obtain the following relations for the population densities:

$$
n_{\mathrm{or}}=n_{\mathrm{or}, \max }\left[1-\frac{d\left[K_{\mathrm{or}}+s_{\mathrm{o}}\right]}{v_{\mathrm{or}} s_{\mathrm{o}}}\right]
$$


and

$$
n_{\mathrm{ro}}=n_{\mathrm{ro}, \max }\left[1-\frac{d\left[K_{\mathrm{ro}}+s_{\mathrm{r}}\right]}{v_{\mathrm{ro}} s_{\mathrm{r}}}\right] .
$$

Defining $s_{\text {tot }}=s_{\mathrm{o}}+s_{\mathrm{r}}$ and using the fact that $n_{\mathrm{or}}=n_{\mathrm{ro}}$, we can then write

$$
n_{\mathrm{ro}, \max }\left[1-\frac{d\left[K_{\mathrm{ro}}+s_{\mathrm{tot}}-s_{\mathrm{o}}\right]}{v_{\mathrm{ro}}\left(s_{\mathrm{tot}}-s_{\mathrm{o}}\right)}\right]=n_{\mathrm{or}, \max }\left[1-\frac{d\left[K_{\mathrm{or}}+s_{\mathrm{o}}\right]}{v_{\mathrm{or}} s_{\mathrm{o}}}\right] .
$$

We now define a parameter $\xi=n_{\mathrm{ro}, \max } / n_{\mathrm{or}, \max }$ which measures the ratio of the maximal population densities, allowing us to rewrite Eq. (S8):

$$
\xi\left[1-\frac{d\left[K_{\mathrm{ro}}+s_{\mathrm{tot}}-s_{\mathrm{o}}\right]}{v_{\mathrm{ro}}\left(s_{\mathrm{tot}}-s_{\mathrm{o}}\right)}\right]=\left[1-\frac{d\left[K_{\mathrm{or}}+s_{\mathrm{o}}\right]}{v_{\mathrm{or}} s_{\mathrm{o}}}\right],
$$

which, upon rearranging, gives a quadratic equation for $s_{\mathrm{O}}$ :

$$
\begin{aligned}
& s_{\mathrm{o}}^{2}\left[\xi v_{\mathrm{or}} v_{\mathrm{ro}}-d \xi v_{\mathrm{or}}-v_{\mathrm{or}} v_{\mathrm{ro}}+d v_{\mathrm{ro}}\right] \\
& +s_{\mathrm{o}}\left[-\xi v_{\mathrm{ro}} s_{\mathrm{tot}} v_{\mathrm{or}}+d \xi v_{\mathrm{or}}\left(K_{\mathrm{ro}}+s_{\mathrm{tot}}\right)+v_{\mathrm{or}} v_{\mathrm{ro}} s_{\mathrm{tot}}+d K_{\mathrm{or}} v_{\mathrm{ro}}-d v_{\mathrm{ro}} s_{\mathrm{tot}}\right]-d K_{\mathrm{or}} v_{\mathrm{ro}} s_{\mathrm{tot}}=0 .
\end{aligned}
$$

Defining the new parameter combinations $\alpha=v_{\text {ro }} / v_{\text {or }}$ and $\omega=(\xi-1) v_{\text {ro }}$, we can rewrite Eq. (S10) as

$$
s_{\mathrm{o}}^{2}[\omega+d(\alpha-\xi)]+s_{\mathrm{o}}\left[d \xi\left(K_{\mathrm{ro}}+s_{\text {tot }}\right)+d \alpha\left(K_{\mathrm{or}}-s_{\text {tot }}\right)-\omega s_{\text {tot }}\right]-d K_{\mathrm{or}} s_{\text {tot }} \alpha=0
$$

This equation has the steady-state solution

$$
s_{\mathrm{o}}=\frac{-B-\sqrt{B^{2}+4 d K_{\mathrm{or}} s_{\mathrm{tot}} \alpha[\omega+d(\alpha-\xi)]}}{2[\omega+d(\alpha-\xi)]}
$$

where

$$
B=d \xi\left(K_{\text {ro }}+s_{\text {tot }}\right)+d \alpha\left(K_{\text {or }}-s_{\text {tot }}\right)-\omega s_{\text {tot }}
$$

An expression for the steady-state concentration of the reduced chemical species $s_{\mathrm{r}}$ can be obtained from Eq. (S12) using $s_{\mathrm{r}}=s_{\mathrm{tot}}-s_{\mathrm{o}}$. Expressions for the steady-state population densities $n_{\mathrm{or}}$ and $n_{\mathrm{or}}$ can be calculated by substitution into Eqs. (S6) and (S7). We take the negative solution to the square root in Eq.(S13), as the other root gives a solution where $s_{\mathrm{O}}>s_{\text {tot }}$ which is not physically realistic.

The solution (S12) is not well-defined right at the threshold between oxidized and reduced states, since both its numerator and denominator go to zero at this point (when $\omega+d(\alpha-\xi)=0$ ). However common sense suggests that at the exact threshold point $s_{\mathrm{r}}=s_{\mathrm{O}}=s_{\text {tot }} / 2$, and we make this assumption in Fig. 2 of the main text.

\section{Biotic-abiotic model}

The dynamical equations for the biotic-abiotic model are

$$
\begin{gathered}
n_{\mathrm{or}}^{\dot{ }}=n_{\mathrm{or}}\left(\frac{v_{\mathrm{or}} s_{\mathrm{o}}}{s_{\mathrm{o}}+K_{\mathrm{or}}}\right)\left(1-\frac{n_{\mathrm{or}}}{n_{\mathrm{or}, \max }}\right)-d n_{\mathrm{or}} \\
\dot{s_{\mathrm{o}}}=\frac{v_{\mathrm{a}} s_{\mathrm{r}}}{s_{\mathrm{r}}+K_{\mathrm{a}}}-\frac{\gamma v_{\mathrm{or}} n_{\mathrm{or}} s_{\mathrm{o}}}{s_{\mathrm{o}}+K_{\mathrm{or}}}\left(1-\frac{n_{\mathrm{or}}}{n_{\mathrm{or}, \max }}\right)
\end{gathered}
$$

and

$$
\dot{s_{\mathrm{r}}}=-\dot{s_{\mathrm{O}}}
$$


These equations correspond to Eqs. (1), (4), (6) and (7) of the main text. Eq. (S14) for the dynamics of the microbial reducer population is exactly the same as in the fully biotic cycle (Eq. S1). However, in this model there is no microbial oxidizer population; rather the oxidation step occurs abiotically and is described by the first term in Eq. (S15). Here $v_{\mathrm{a}}$ is the maximal reaction rate of the abiotic oxidation step while $K_{\mathrm{a}}$ is the concentration of $\mathrm{s}_{\mathrm{r}}$ at which the rate is half-maximal. The case of a spontaneous reaction (not catalysed) can be modelled by setting $K_{\mathrm{a}}$ to be very large.

Setting $n_{\mathrm{or}}=0$ in Eq. (S14) we obtain, as for the fully biotic cycle

$$
d=\left(\frac{v_{\mathrm{or}} s_{\mathrm{o}}}{s_{\mathrm{o}}+K_{\mathrm{or}}}\right)\left(1-\frac{n_{\mathrm{or}}}{n_{\mathrm{or}, \max }}\right)
$$

Setting $\dot{s_{\mathrm{O}}}=0$ in Eq. (S15) gives:

$$
\frac{v_{\mathrm{a}} s_{\mathrm{r}}}{K_{\mathrm{a}}+s_{\mathrm{r}}}=\frac{\gamma v_{\mathrm{or}} n_{\mathrm{or}} s_{\mathrm{o}}}{K_{\mathrm{or}}+s_{\mathrm{o}}}\left[1-\frac{n_{\mathrm{or}}}{n_{\mathrm{or}, \max }}\right] .
$$

Combining Eqs (S17) and (S18) gives:

$$
\frac{v_{\mathrm{a}} s_{\mathrm{r}}}{K_{\mathrm{a}}+s_{\mathrm{r}}}=\gamma n_{\mathrm{or}} d=\gamma d n_{\mathrm{or}, \max }\left[1-\frac{d\left[K_{\mathrm{or}}+s_{\mathrm{o}}\right]}{v_{\mathrm{or}} s_{\mathrm{o}}}\right] .
$$

We now use the fact that $s_{\mathrm{r}}=s_{\text {tot }}-s_{\mathrm{o}}$, and define a new parameter combination $\phi=\gamma d n_{\mathrm{or}, \max }$, to obtain

$$
\frac{v_{\mathrm{a}}\left(s_{\text {tot }}-s_{\mathrm{o}}\right)}{K_{\mathrm{a}}+\left(s_{\text {tot }}-s_{\mathrm{o}}\right)}=\phi-\frac{\phi d\left[K_{\mathrm{or}}+s_{\mathrm{o}}\right]}{v_{\mathrm{or}} s_{\mathrm{o}}} .
$$

Rearranging Eq. (S20) produces a quadratic equation for $s_{\mathrm{O}}$ :

$$
s_{\mathrm{o}}^{2}\left[\phi v_{\mathrm{or}}-\phi d-v_{\mathrm{or}} v_{\mathrm{a}}\right]+s_{\mathrm{o}}\left[\phi d\left(K_{\mathrm{a}}+s_{\mathrm{tot}}\right)-\phi v_{\mathrm{or}}\left(K_{\mathrm{a}}+s_{\mathrm{tot}}\right)-v_{\mathrm{or}} v_{\mathrm{a}} s_{\text {tot }}-\phi d K_{\mathrm{or}}\right]+\phi d K_{\mathrm{or}} K_{\mathrm{a}}+\phi d K_{\mathrm{or}} s_{\text {tot }}=0
$$

the solution of which is

$$
s_{\mathrm{o}}=\frac{-B^{\prime}-\sqrt{B^{\prime 2}-4\left(\phi v_{\mathrm{or}}-\phi d-v_{\mathrm{or}} v_{\mathrm{a}}\right)\left(K_{\mathrm{or}} d \phi\left(K_{\mathrm{a}}+s_{\mathrm{tot}}\right)\right)}}{2\left(\phi v_{\mathrm{or}}-\phi d-v_{\mathrm{or}} v_{\mathrm{a}}\right)}
$$

where $B^{\prime}$ is

$$
B^{\prime}=\phi d\left(K_{\mathrm{a}}+s_{\text {tot }}\right)-\phi v_{\text {or }}\left(K_{\mathrm{a}}+s_{\text {tot }}\right)-v_{\text {or }} v_{\mathrm{a}} s_{\text {tot }}-\phi d K_{\text {or }}
$$

Again, we take the negative solution to the square root, as the other root gives a solution where $s_{\mathrm{O}}>s_{\text {tot }}$ which is not physically realistic. An expression for $s_{\mathrm{r}}$ can be calculated from Eq. (S22) using $s_{\mathrm{r}}=s_{\text {tot }}-s_{\mathrm{O}}$. An expression for the density $n_{\text {or }}$ of the reducer population can be obtained by subtituting Eq. (S22) into Eq. (S17).

\section{Abiotic-abiotic cycles}

In this study, we have not analyzed in detail the case of a redox cycle where both the oxidation and reduction steps are abiotic. This is because abiotic reduction steps are rare in the natural environment, and we are primarly interested in the effects of microbial population dynamics. However, equations for an entirely abiotic cycle can easily be constructed along the same lines. These equations turn out to be exactly equivalent to those of the Goldbeter-Koshland model for enzymatic phosphorylation-dephosphorylation cycles [1] (see section S7). If both reactions are catalyzed and can be saturated with respect to their chemical substrates, then redox regime shifts are predicted in this case. 


\section{S2. PREDICTIONS FOR THE MICROBIAL POPULATION DENSITY}

In Fig. 2 of the main text, we show how the global redox state of our model systems, $s_{\mathrm{o}} / s_{\text {tot }}$, changes with the degree of reductive or oxidative driving. This data reveals sharp transitions (redox regime shifts), the sharpness of which increases with $s_{\text {tot }}$. Here we discuss how the microbial population densities vary during these transitions. Fig. S1 shows the density of the reducing microbial population, $n_{\mathrm{or}}$ (which for the fully biotic cycle is equal to $n_{\text {ro }}$ ) as a function of the parameters which form proxies for reductive or oxidative driving $\left(v_{\text {or }}, v_{\text {ro }}\right.$ and $\left.v_{\mathrm{a}}\right)$. In all cases, the microbial population density responds gradually to changes in reductive or oxidative driving, even when the global ecosystem redox state responds sharply (compare to Fig. 2 of the main text). As the driving increases, the population responds by increasing in size, until it approaches its maximum density $n_{\max }$ which in this case is equal to $9 \times 10^{7}$ cells/l. For the fully biotic cycle, and for the biotic-abiotic cycle under reductive driving, the steady-state population size is only weakly dependent on the total concentration of the element being cycled $\left(s_{t o t}\right)$.

\section{Fully biotic cycle}

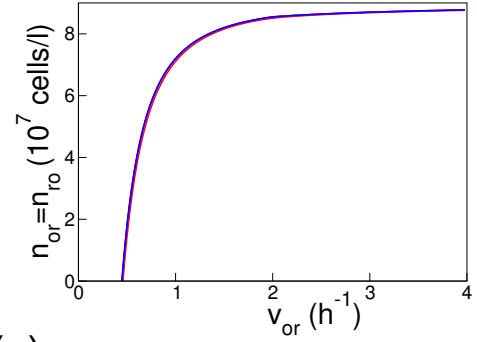

(a)

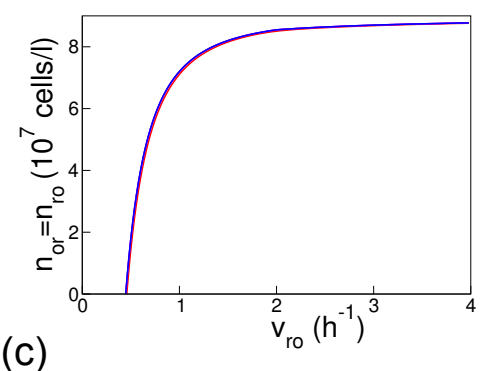

Biotic-abiotic cycle

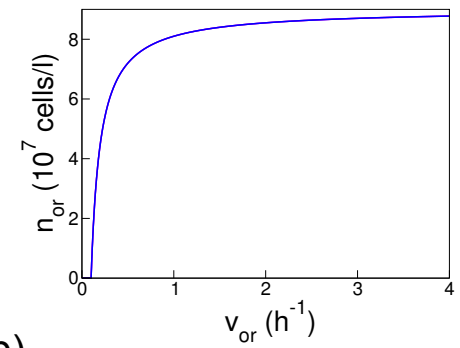

(b)

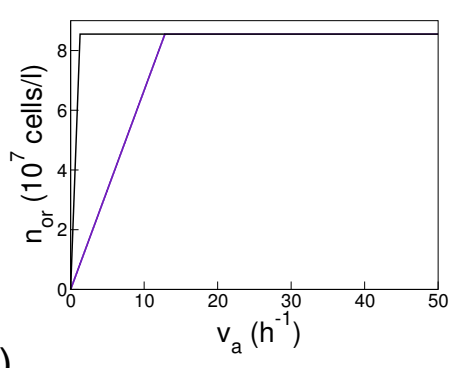

(d)

Figure S1: Changes in the microbial population density as the model undergoes a redox regime shift. For the fully biotic cycle (left panels), the oxidizing and reducing populations have equal population densities, $n_{\text {or }}=n_{\text {ro }}$. For the biotic-abiotic cycle (right panels), the population density of the reducing population, $n_{\mathrm{or}}$, is plotted. The top panels show the response to increase in reductive driving (mimicking increase in acetate) while the bottom panels show the response to an increase in oxidative driving (mimicking increase in oxygen). The parameters are as in Fig. 2 of the main text: $K_{\text {or }}=K_{\text {ro }}=K_{\mathrm{a}}=1 \mu \mathrm{M}$, $n_{\text {or,max }}=n_{\text {ro,max }}=9 \times 10^{7}$ cells $/ 1, d=0.1 \mathrm{~h}^{-1}$ and $\gamma=3 \times 10^{-8} \mu$ moles/cell. In a, $v_{\text {ro }}$ is fixed at $2 \mathrm{~h}^{-1}$, in $\mathrm{b}$ and $\mathrm{d} v_{\text {or }}$ is fixed at $2 \mathrm{~h}^{-1}$ and in c, $v_{\mathrm{a}}=0.2 \mu \mathrm{Mh}^{-1}$. Different colors represent different values of $s_{\text {tot }}$, as in Fig. 2 of the main text. Where the analytical solution of our model equations predicts a negative population density, we have set $n_{\mathrm{or}}=n_{\mathrm{ro}}=0$. 


\section{S3. THE ROLE OF THE MAXIMAL POPULATION DENSITY PARAMETER $n_{\max }$}

In our model, we represent the fact that the microbial population density is ultimately limited by factors other than the chemical being cycled $(s)$, by introducing a "logistic" term $\left(1-n / n_{\max }\right)$ in the equation for the microbial growth kinetics. Here we explore the effect of varying the parameters $n_{\text {or,max }}$ and $n_{\text {ro,max }}$ corresponding to the reducer and oxidizer populations, and find that the qualitative steady-state behaviour of the model is unaffected by these parameters, although the time-scale for reaching the steady state is affected. In the next section we will also show that similar results are obtained for a model where we replace this term by equations that represent explicit growth-limitation by an additional nutrient source.

\section{Fully biotic cycle}

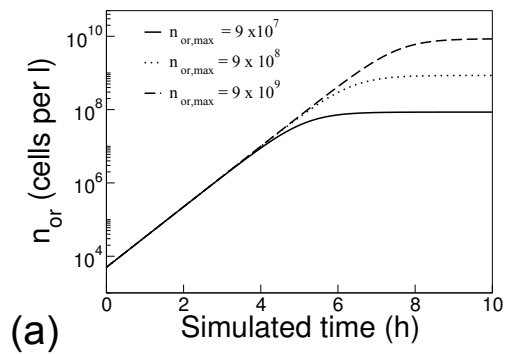

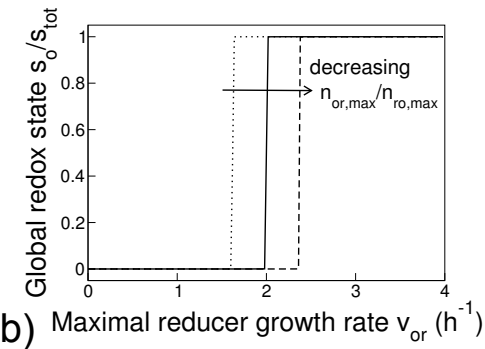

(b)

\section{Biotic-abiotic cycle}

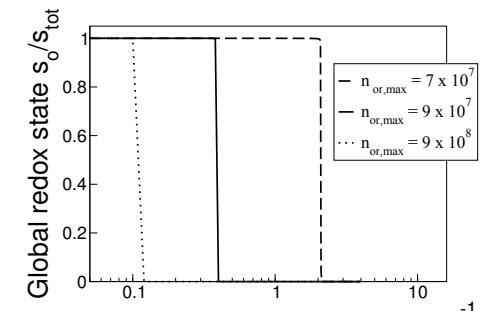

(c)

Figure S2: The role of the maximal population density parameter. Panel (a) shows that the time to reach the steady state increases as $n_{\text {or,max }}$ increases, for the fully biotic cycle. Panel (b), also for the fully biotic cycle, shows that changing the ratio of maximal population sizes shifts the tipping point at which the redox regime shift happens but does not alter the qualitative behaviour of the model. Panel (c), for the biotic-abiotic cycle, shows that changing the maximal microbial population density $n_{\text {or,max }}$ again shifts the tipping point, but redox regime shifts are still present. For this case, if $n_{\text {or,max }}$ becomes too small the regime shift is lost (the system remains oxic for all values of $v_{\text {or }}$ ). The parameters are as in Fig. 2 of the main text: $K_{\text {or }}=K_{\text {ro }}=K_{\mathrm{a}}=1 \mu \mathrm{M}, d=0.1 \mathrm{~h}^{-1}, \gamma=3 \times 10^{-8} \mu$ moles $/$ cell and $s_{\text {tot }}=0.2 \mathrm{M}$. In panel c, $v_{\mathrm{a}}=0.2 \mu \mathrm{Mh}^{-1}$.

\section{Fully biotic cycle}

In our fully biotic model, the steady state solution (Eqs. (S12) and (S13)) depends only on the ratio of the maximal population densities of the reducer and oxidizer populations, $\xi=n_{\mathrm{or}, \max } / n_{\mathrm{ro} \text {, max }}$, and not on the absolute values of $n_{\mathrm{or}, \max }$ and $n_{\mathrm{ro}, \max }$. However, the timescale at which the steady state is reached does depend on the absolute maximal population densities; as these increase the system responds more slowly (Fig. S2a). Considering only the steady-state solution, changing the ratio of maximal population densities $\xi$ alters the tipping point at which the redox regime shift is predicted to occur in our model (Fig. S2b) but does not change the qualitative behaviour of the model.

\section{Biotic-abiotic cycle}

For the biotic-abiotic model, the steady-state solution (Eqs. (S22) and (S23)) does depend on the maximal population density of the reducer population, $n_{\mathrm{or} \text { max }}$. Upon varying this parameter (Fig. S2c) we find that the tipping point at which the redox regime shift happens changes; for a smaller value of $n_{\text {or,max }}$, we require a greater value of the maximal reducer growth rate $v_{\text {or }}$ to trigger the regime shift. In fact, in this model, the regime shift is lost altogether if the maximal microbial population density is too small. To observe regime shifts, we require that $v_{\mathrm{a}}<\gamma d n_{\mathrm{or}, \mathrm{max}}$. This is because, at steady state, the maximal microbial "conversion rate" of $\mathrm{s}_{\mathrm{o}}$ to $\mathrm{s}_{\mathrm{r}}$ is given by $\gamma d n_{\mathrm{or}, \max }$ while the maximal abiotic conversion rate of $\mathrm{s}_{\mathrm{r}}$ to $\mathrm{s}_{\mathrm{o}}$ is set by $v_{\mathrm{a}}$. For a regime shift to occur, the biotic conversion rate must be able to exceed the abiotic conversion rate. 


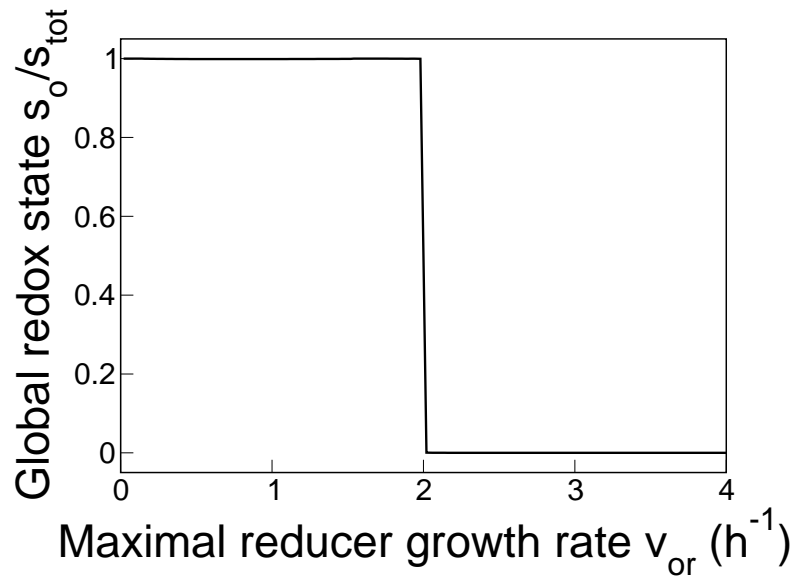

Figure S3: Redox regime shifts are still observed in a model where the term $\left(1-n(t) / n_{\max }\right)$ is replaced by explicit growth limitation by a supply of an external nutrient. The results show the redox state of the ecosystem $\left(s_{\circ} / s_{\text {tot }}\right)$ as a function of the maximal growth rate of the reducer population $\left(v_{\mathrm{or}}\right)$. The data was obtained by numerical solution to steady state of Eqs. (S24)-(S28), for the parameter set $v_{\mathrm{ro}}=2 \mathrm{~h}^{-1}, \gamma=3 \times 10^{-8} \mu$ moles $/$ cell, $K_{\mathrm{ro}}=K_{\mathrm{or}}=K_{\mathrm{x}}=1 \mu \mathrm{M}, s_{\mathrm{tot}}=1 \mathrm{M}, d=0.1 \mathrm{~h}^{-1}$ and $b=0.001 \mathrm{Mh}^{-1}$.

In this section, we replace the population density limitation term $\left(1-n(t) / n_{\max }\right)$, used in the main text, with an explicit model for growth limitation by an external nutrient source. We show that this model still reproduces the regime shifting phenomenon as we vary the availability of acetate. To represent limitation by an external nutrient we introduce additional differential equations for the concentration $x(t)$ of this nutrient into our model for the fully biotic cycle. We suppose that the nutrient is supplied at a fixed rate $b$ and is consumed by both the oxidiser and reducer populations as they grow. Both microbial growth rates are assumed to depend on $x(t)$ via multiplicative Monod growth terms (with half-saturation constant $K_{\mathrm{x}}$ ). This results in the following set of equations:

$$
\begin{gathered}
\frac{d x(t)}{d t}=b-\gamma\left(\frac{x}{K_{\mathrm{x}}+x}\right)\left[\left(\frac{n_{\mathrm{ro}} v_{\mathrm{ro}} s_{\mathrm{r}}}{K_{\mathrm{ro}}+s_{\mathrm{r}}}\right)+\left(\frac{n_{\mathrm{or}} v_{\mathrm{or}} s_{o}}{K_{\mathrm{or}}+s_{\mathrm{o}}}\right)\right] \\
\frac{d n_{\mathrm{ro}}(t)}{d t}=\frac{v_{\mathrm{ro}} n_{\mathrm{ro}}(t) s_{\mathrm{r}}(t)}{K_{\mathrm{ro}}+s_{\mathrm{r}}(t)}\left(\frac{x}{K_{\mathrm{x}}+x}\right)-d n_{\mathrm{ro}}(t) \\
\frac{d n_{\mathrm{or}}(t)}{d t}=\frac{v_{\mathrm{or}} n_{\mathrm{or}}(t) s_{\mathrm{o}}(t)}{K_{\mathrm{or}}+s_{\mathrm{o}}(t)}\left(\frac{x}{K_{\mathrm{x}}+x}\right)-d n_{\mathrm{or}}(t) \\
\frac{d s_{\mathrm{r}}(t)}{d t}=-\frac{\gamma v_{\mathrm{ro}} n_{\mathrm{ro}}(t) s_{\mathrm{r}}(t)}{K_{\mathrm{ro}}+s_{\mathrm{r}}(t)}\left(\frac{x}{K_{\mathrm{x}}+x}\right)+\frac{\gamma v_{\mathrm{or}} n_{\mathrm{or}}(t) s_{\mathrm{o}}(t)}{K_{\mathrm{or}}+s_{\mathrm{o}}(t)}\left(\frac{x}{K_{\mathrm{x}}+x}\right) \\
\frac{d s_{\mathrm{o}}(t)}{d t}=-\frac{d s_{\mathrm{r}}(t)}{d t}
\end{gathered}
$$

These equations were iterated numerically using the Euler-forward method to compute the steady state, for the parameter set $v_{\mathrm{ro}}=2 \mathrm{~h}^{-1}, \gamma=3 \times 10^{-8} \mu$ moles $/$ cell, $K_{\mathrm{ro}}=K_{\mathrm{or}}=K_{\mathrm{x}}=1 \mu \mathrm{M}, s_{\mathrm{tot}}=1 \mathrm{M}, d=0.1 \mathrm{~h}-1$ and $b=0.001 \mathrm{Mh}^{-1}$. Fig. S3 shows the resulting prediction for the redox state of the ecosystem $\left(s_{\mathrm{o}} / s_{\text {tot }}\right)$ as a function of the maximal growth rate of the reducer population $\left(v_{\mathrm{or}}\right.$, which serves as a proxy for the availability of acetate). Comparing with Fig. 2a of the main text, we see that indeed this extended model does produce a redox regime shift which is qualitatively similar to that of the model discussed in the main text. 
S5. SPATIAL HETEROGENEITY ACCENTUATES REDOX REGIME SHIFTS

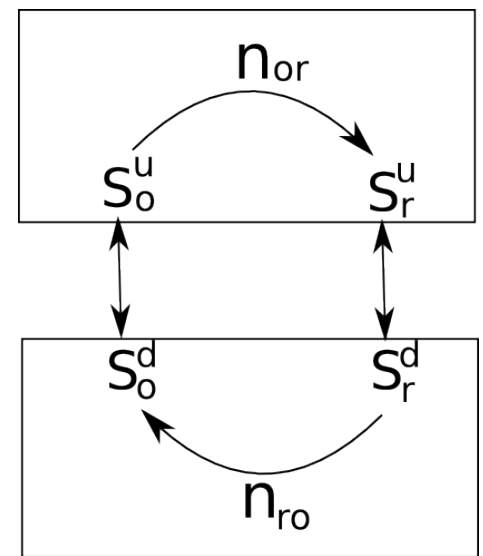

Figure S4: The "two population, two box" model. The oxidizing and reducing microbial populations are shown by $n_{\mathrm{ro}}$ and $n_{\text {or }}$, while the oxidized and reduced forms of chemical species s are denoted $s_{\mathrm{o}}$ and $s_{\mathrm{r}}$. The superscripts $\mathrm{u}$ and $\mathrm{d}$ refer to the upper and lower boxes respectively. The double-headed arrows denote chemical diffusion.

In the main text, we showed analytically that redox regime shifts can occur in response to changes in oxygen or acetate availability, for a "well-mixed" model with two microbial populations (reducers and oxidizers). In reality, however, microbial reduction and oxidation processes are usually spatially separated. In this section, we show that redox regime shifting is in fact enhanced for a model which captures this spatial separation.

We extend the basic "two-population" model of the main text, for the fully biotic cycle, by introducing two spatial boxes, coupled by chemical diffusion. We assume that the reducer population is located in the lower box while the oxidizer population is located in the upper box. The reduced and oxidized chemical species $s_{\mathrm{r}}$ and $s_{\mathrm{O}}$ can be located in either box and are transferred between boxes by diffusion, as shown in Fig. S4. For the chemical species, we use the superscripts $\mathrm{u}$ and $\mathrm{d}$ to denote the upper and lower boxes respectively.

This "two population, two box model" is described by the following dynamical equations:

$$
\begin{gathered}
\frac{d n_{\mathrm{or}}(t)}{d t}=\frac{v_{\mathrm{or}} n_{\mathrm{or}}(t) s_{\mathrm{o}}^{\mathrm{u}}(t)}{K_{\mathrm{or}}+s_{\mathrm{o}}^{\mathrm{u}}(t)}\left(1-\frac{n_{\mathrm{or}}(t)}{n_{\mathrm{or}, \max }}\right)-d n_{\mathrm{or}}(t) \\
\frac{d n_{\mathrm{ro}}(t)}{d t}=\frac{v_{\mathrm{ro}} n_{\mathrm{ro}}(t) s_{\mathrm{r}}^{\mathrm{d}}(t)}{K_{\mathrm{ro}}+s_{\mathrm{r}}^{\mathrm{d}}(t)}\left(1-\frac{n_{\mathrm{ro}}(t)}{n_{\mathrm{ro}, \max }}\right)-d n_{\mathrm{ro}}(t) \\
\frac{d s_{\mathrm{o}}^{\mathrm{u}}(t)}{d t}=-\frac{\gamma v_{\mathrm{or}} n_{\mathrm{or}}(t) s_{\mathrm{o}}^{\mathrm{u}}(t)}{K_{\mathrm{or}}+s_{\mathrm{o}}^{\mathrm{u}}(t)}\left(1-\frac{n_{\mathrm{or}}(t)}{n_{\mathrm{or}, \max }}\right)-k s_{\mathrm{o}}^{\mathrm{u}}(t)+k s_{\mathrm{o}}^{\mathrm{d}}(t) \\
\frac{d s_{\mathrm{o}}^{\mathrm{d}}(t)}{d t}=\frac{\gamma v_{\mathrm{ro}} n_{\mathrm{ro}}(t) s_{\mathrm{r}}^{\mathrm{d}}(t)}{K_{\mathrm{ro}}+s_{\mathrm{r}}^{\mathrm{d}}(t)}\left(1-\frac{n_{\mathrm{ro}}(t)}{n_{\mathrm{ro}, \max }}\right)-k s_{\mathrm{o}}^{\mathrm{d}}(t)+k s_{\mathrm{o}}^{\mathrm{u}}(t) \\
\frac{d s_{\mathrm{r}}^{\mathrm{u}}(t)}{d t}=\frac{\gamma v_{\mathrm{or}} n_{\mathrm{or}}(t) s_{\mathrm{o}}^{\mathrm{u}}(t)}{K_{\mathrm{or}}+s_{\mathrm{o}}^{\mathrm{u}}(t)}\left(1-\frac{n_{\mathrm{or}}(t)}{n_{\mathrm{or}, \max }}\right)-k s_{\mathrm{r}}^{\mathrm{u}}(t)+k s_{\mathrm{r}}^{\mathrm{d}}(t) \\
\frac{d s_{\mathrm{r}}^{\mathrm{d}}(t)}{d t}=-\frac{\gamma v_{\mathrm{or}} n_{\mathrm{or}}(t) s_{\mathrm{o}}^{\mathrm{u}}(t)}{K_{\mathrm{or}}+s_{\mathrm{o}}^{\mathrm{u}}(t)}\left(1-\frac{n_{\mathrm{or}}(t)}{n_{\mathrm{or}, \max }}\right)-k s_{\mathrm{r}}^{\mathrm{d}}(t)+k s_{\mathrm{r}}^{\mathrm{u}}(t)
\end{gathered}
$$


Here, as before, $K_{\text {or }}$ and $K_{\text {ro }}$ are the half-saturation constants for the microbial reducers and oxidizers respectively, $v_{\text {or }}$ and $v_{\text {ro }}$ are their maximal growth rates, $n_{\text {or,max }}$ and $n_{\text {ro,max }}$ are their maximal population sizes, $\gamma$ is the yield parameter (amount of chemical substrate needed to create one bacterium) and $d$ is the microbial death rate. Compared to the main text, we now have a new parameter $k$ which represents the rate of diffusive chemical transport between the two spatial boxes.

To investigate the behaviour of the two-population, two-box model, we integrated Eqs (S29)-(S34) numerically using the Euler-forward method to steady state. Fig. S5 shows that indeed we obtain redox regime shifting behaviour as we vary the parameters $v_{\text {or }}$ or $v_{\text {ro }}$, which serve as proxies for the acetate and oxygen availabilities. Comparing Fig. S5a and b we see that the redox regime shift occurs over a broader range of parameter values as the rate of chemical diffusion $(k)$ increases.
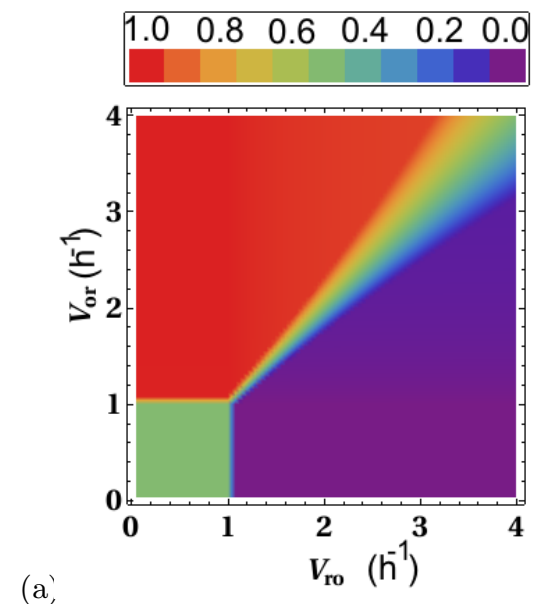

$\begin{array}{llllll}1.0 & 0.8 & 0.6 & 0.4 & 0.2 & 0.0\end{array}$

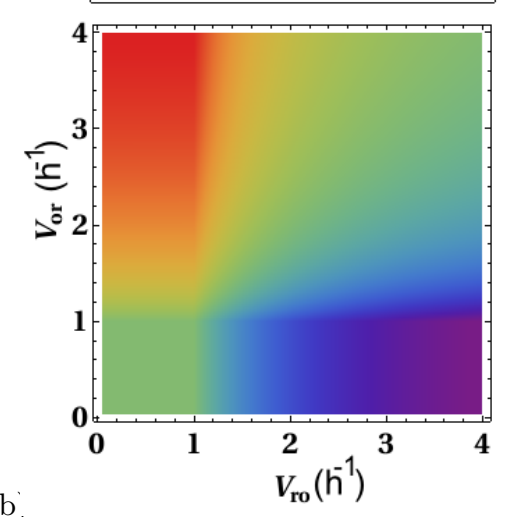

Figure S5: Steady state of the fully biotic spatial heterogeneous model (a) and (b) are phase plots where the colour key refers to the steady state value of $s_{\mathrm{o}} / s_{\text {tot }}$ with parameters $d=0.1 \mathrm{~h}^{-1}, \gamma=3 \times 10^{-8} \mu \mathrm{moles} /$ cell, $n_{\max }=9 \times 10^{7}$ cells $/$ litre, and $K_{\text {ro }}=K_{\text {or }}=K_{\mathrm{M}}=1 \mu \mathrm{M}$ (a): Slow diffusion $k=0.001 \mathrm{~h}^{-1}$. (b): Fast diffusion, $k=100 \mathrm{~h}^{-1}$. 


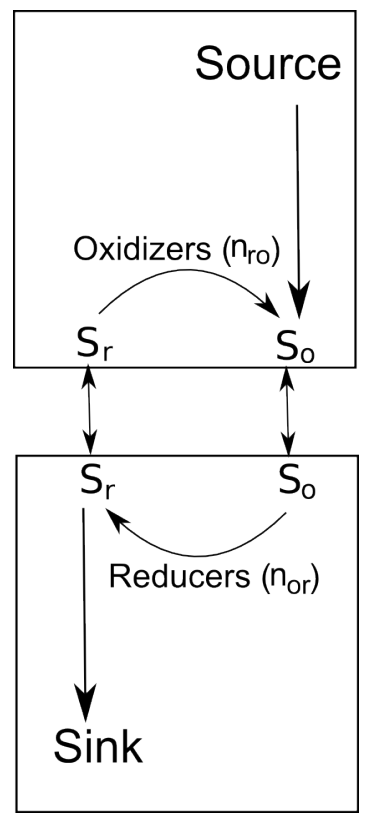

Figure S6: The "two population, two box" model with chemical sources and sinks.

In the natural environment, redox-cycling microbial ecosystems do not exist in isolation but are coupled to the environment via chemical sources and sinks. For example, in the sulphur cycle, hydrogen sulphide can react with iron to form pyrite, which is sufficiently unreactive that it effectively represents a loss of sulphide [2]. To test the effect of chemical sources and sinks on the behaviour of our models, we simulated the model shown in Fig. S6, in which the "two-population, two-box model" of section S5 is supplemented by terms describing the inflow of the oxidized chemical species $\mathrm{s}_{\mathrm{O}}$ and the outflow of the reduced chemical species $\mathrm{s}_{\mathrm{r}}$. This topology was chosen to roughly correspond to the biogeochemical sulfur cycle, where oxidized substrate (sulfate) is input from rivers, and reduced substrate (sulfide) reacts with iron oxides to form pyrite. We expect other topologies to produce similar results.

The differential equations describing this model are

$$
\begin{gathered}
\frac{d n_{\mathrm{or}}(t)}{d t}=\frac{v_{\mathrm{or}} n_{\mathrm{or}}(t) s_{\mathrm{o}}^{\mathrm{u}}(t)}{K_{\mathrm{or}}+s_{\mathrm{o}}^{\mathrm{u}}(t)}\left(1-\frac{n_{\mathrm{or}}(t)}{n_{\mathrm{or}, \max }}\right)-d n_{\mathrm{or}}(t) \\
\frac{d n_{\mathrm{ro}}(t)}{d t}=\frac{v_{\mathrm{ro}} n_{\mathrm{ro}}(t) s_{\mathrm{r}}^{\mathrm{d}}(t)}{K_{\mathrm{ro}}+s_{\mathrm{r}}^{\mathrm{d}}(t)}\left(1-\frac{n_{\mathrm{ro}}(t)}{n_{\mathrm{ro}, \max }}\right)-d n_{\mathrm{ro}}(t) \\
\frac{d s_{\mathrm{o}}^{\mathrm{u}}(t)}{d t}=-\frac{\gamma v_{\mathrm{or}} n_{\mathrm{or}}(t) s_{\mathrm{o}}^{\mathrm{u}}(t)}{K_{\mathrm{or}}+s_{\mathrm{o}}^{\mathrm{u}}(t)}\left(1-\frac{n_{\mathrm{or}}(t)}{n_{\mathrm{or}, \max }}\right)-k s_{\mathrm{o}}^{\mathrm{u}}(t)+k s_{\mathrm{o}}^{\mathrm{d}}(t)+J \\
\frac{d s_{\mathrm{o}}^{\mathrm{d}}(t)}{d t}=\frac{\gamma v_{\mathrm{ro}} n_{\mathrm{ro}}(t) s_{\mathrm{r}}^{\mathrm{d}}(t)}{K_{\mathrm{ro}}+s_{\mathrm{r}}^{\mathrm{d}}(t)}\left(1-\frac{n_{\mathrm{ro}}(t)}{n_{\mathrm{ro}, \max }}\right)-k s_{\mathrm{o}}^{\mathrm{d}}(t)+k s_{\mathrm{o}}^{\mathrm{u}}(t) \\
\frac{d s_{\mathrm{r}}^{\mathrm{u}}(t)}{d t}=\frac{\gamma v_{\mathrm{or}} n_{\mathrm{or}}(t) s_{\mathrm{o}}^{\mathrm{u}}(t)}{K_{\mathrm{or}}+s_{\mathrm{o}}^{\mathrm{u}}(t)}\left(1-\frac{n_{\mathrm{or}}(t)}{n_{\mathrm{or}, \max }}\right)-k s_{\mathrm{r}}^{\mathrm{u}}(t)+k s_{\mathrm{r}}^{\mathrm{d}}(t)
\end{gathered}
$$


and

$$
\frac{d s_{\mathrm{r}}^{\mathrm{d}}(t)}{d t}=-\frac{\gamma v_{\mathrm{or}} n_{\mathrm{or}}(t) s_{\mathrm{o}}^{\mathrm{u}}(t)}{K_{\mathrm{or}}+s_{\mathrm{o}}^{\mathrm{u}}(t)}\left(1-\frac{n_{\mathrm{or}}(t)}{n_{\mathrm{or}, \max }}\right)-k s_{\mathrm{r}}^{\mathrm{d}}(t)+k s_{\mathrm{r}}^{\mathrm{u}}(t)-I s_{\mathrm{r}}^{\mathrm{d}}(t) .
$$

Eqs. (S35) - (S40) are identical to Eqs. (S29)-(S34) except for the addition of the source and sink terms in Eqs. (S37) and (S40). Here $J$ represents an inflow rate (in units of $\mu \mathrm{Mh}^{-1}$ ) while $I$ represents an outflow rate (in units of $\left.\mathrm{h}^{-1}\right)$.

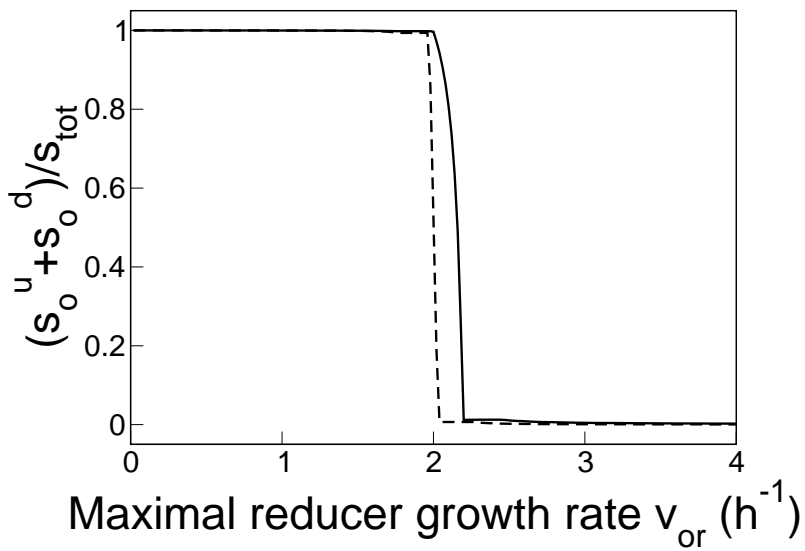

Figure S7: Redox regime shifts with source and sink terms included. The parameters are $K_{\mathrm{ro}}=K_{\mathrm{or}}=1 \mu \mathrm{M}, s_{\mathrm{tot}}=4 \mathrm{M}$, $n_{\text {or, } \max }=n_{\text {ro, } \max }=9 \times 10^{7}$ cells per litre, $\gamma=3 \times 10^{-8} \mu$ moles $/$ cell, $k=10 \mathrm{~h}^{-1}, d=0.1 \mathrm{~h}^{-1}$ and $v_{\text {ro }}=2 \mathrm{~h}^{-1}$. The black solid line shows results for $I=1 \mathrm{~h}^{-1}$ and $J=1 \mathrm{mMh}^{-1}$. The black dashed line shows results for $I=1 \times 10^{-3} \mathrm{~h}^{-1}$ and $J=1 \times 10^{-3} \mu \mathrm{Mh}^{-1}$. The black dashed line shows equivalent results for $I=1 \times 10^{-5} \mathrm{~h}^{-1}$ and $J=10 \mu \mathrm{Mh}^{-1}$

Eqs. (S35) - (S40) were integrated numerically using the Euler-forward method to reach the steady state. Fig. S7 shows the resulting predictions for the global redox state, as measured by the fraction of oxidized chemical species $\left(s_{o}^{\mathrm{u}}+s_{o}^{\mathrm{d}}\right) / s_{\text {tot }}$, as a function of the maximal growth rate of the reducer population, $v_{\text {or }}$, which serves as a proxy for the availability of acetate. We still observe redox regime shifts in the presence of the source and sink terms. 


\section{S7. CONNECTION BETWEEN OUR MODEL AND THE GOLDBETER-KOSHLAND MODEL FOR ENZYMATIC PHOSPHORYLATION-DEPHOSPHORYLATION CYCLES}

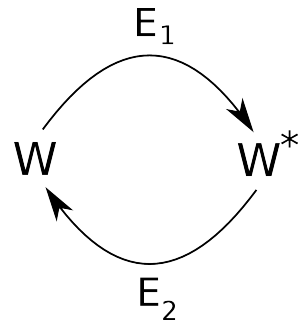

Figure S8: The model of Goldbeter and Koshland for an enzymatic phosphorylation-dephosphorylation cycle. here $\mathrm{E}_{1}$ represents a kinase enzyme, $\mathrm{E}_{2}$ represents a phosphatase enzyme, $\mathrm{W}$ represents the unphosphorylated form of a protein and $\mathrm{W}^{*}$ represents its phosphorylated form [1].

An interesting mathematical analogy exists between the microbial nutrient-cycling models introduced in our work and classic results obtained by Goldbeter and Koshland for a phenomenon on a completely different scale, relating to the biochemical networks that control the response of a single biological cell to an external stimulus. Cells often mediate metabolic responses to stimuli such as changes in temperature or nutrient concentration, using phosphorylation-dephosphorylation cycles $[3,4]$. In these cycles, a target protein is activated by addition of a phosphate group, and deactivated by removal of the phosphate group; the kinase and phosphatase enzymes mediating these reactions act in opposition to each other, the kinase being stimulated by the extracellular signal (Fig. S8). Activation of the target enzyme leads ultimately to the cell's response. In this model, the "input signal" is mediated via a change in the relative activities of the kinase and phosphatase enzymes, while the "output response" is manifest as a change in the relative proportions of the target protein $W$ which are in the phosphorylated and dephosphorylated forms.

Assuming Michaelis-Menten kinetics for the phosphorylation and dephosphorylation steps, Goldbeter and Koshland derived steady-state solutions for the concentration of protein substrate $W$ in the dephosphorylated form $([W])$. These results revealed a phenomenon which Goldbeter and Koshland termed "zero order ultrasensitivity": when the total substrate concentration $W_{\text {tot }}$ is high, the system responds extremely sharply to a small change in the relative activities of the kinase and phosphatase enzymes $[1,4]$.

Goldbeter and Koshland's steady state solution is given by

$$
[W]=\frac{-B-\sqrt{B^{2}-4\left[W_{\text {tot }}\right]\left[E_{2}\right] K_{\mathrm{M}} \alpha\left(\left[E_{1}\right] \alpha-\left[E_{2}\right]\right)}}{2\left(\alpha\left[E_{1}\right]-\left[E_{2}\right]\right)}
$$

where

$$
B=\left[W_{\text {tot }}\right]\left(\left[E_{2}\right]-\alpha\left[E_{1}\right]\right)+K_{\mathrm{M}}\left(\left[E_{2}\right]+\alpha\left[E_{1}\right]\right) .
$$

Here, $\left[E_{1}\right]$ and $\left[E_{2}\right]$ represent the concentrations of the kinase and phosphatase enzymes, $K_{\mathrm{M}}$ is the half-saturation constant for the enzymatic reactions (assumed to be the same for kinase and phosphatase) and $\alpha$ is the ratio of the maximal reaction rates for the kinase and phosphatase.

The two-population, fully biotic, nutrient cycle models studied in our work bear a striking topological similarity to the Goldbeter-Koshland model (compare Fig. 2e of the main text with Fig. S8). In this analogy, the role of the kinase and phosphatase enzymes is played by the reducing and oxidizing microbial populations while the role of the protein target molecule is played by the chemical species being cycled.

This analogy extends to the mathematical solutions of the two models. If we assume that both microbial populations have identical half-saturation constants $\left(K_{\mathrm{ro}}=K_{\mathrm{or}}=K\right)$ and maximal population densities $\left(n_{\mathrm{ro}, \max }=n_{\mathrm{or}, \max }=\right.$ $\left.n_{\max }\right)$, then $\xi=1$ and $\omega=(\xi-1) v_{\mathrm{ro}}=0$. 
We then obtain the following solution for the concentration of the oxidized chemical species (from Eq. S12):

$$
s_{\mathrm{o}}=\frac{-B-\sqrt{B^{2}-4 s_{\mathrm{tot}} K \alpha(\alpha-1)}}{2(\alpha-1)}
$$

where

$$
B=s_{\text {tot }}(1-\alpha)+K(1+\alpha)
$$

and $\alpha=v_{\text {ro }} / v_{\text {or }}$. Comparing Eq. (S43) with Eq. (S41) we see that the two models are mathematically equivalent, as long as the enzyme concentrations in the Goldbeter-Koshland model are set equal $\left(\left[E_{1}\right]=\left[E_{2}\right]\right)$. Note that the maximal population density $n_{\max }$ does not play an analogous role to the enzyme concentration, however. When we set the two maximal population densities to be different, we obtain the more complex steady state solution shown in Eq. (S12).

It is important to note that this mathematical analogy does not extend to the biotic-abiotic cycle model, which has a more complex steady state solution, Eq. (S22), although with a similar functional form. 


\section{S8. THE FOUR-POPULATION TWO-BOX MODEL: EXPLICIT GENERATION OF ACETATE AND OXYGEN}

In the main text, we present results for an extended redox cycling ecosystem model in which the populations of photosynthesizers and organic matter degraders are explicitly represented, as well as the reducers and oxidizers. This model accounts for the production of acetate and oxygen, having as its inputs the light intensity (which stimulates production of oxygen by the photosynthesizer population) and the organic matter concentration (which stimulates production of acetate by the degrader population). This model is illustrated in the main text, Fig. 3a, for the fully biotic cycle. Here we list the equations corresponding to this model, which were solved numerically to obtain the data shown in Fig. 3b of the main text, and give the full set of parameters used in these calculations. We also discuss an equivalent model for the biotic-abiotic cycle.

Equations and parameters for the fully biotic four-population, two-box model

The equations corresponding to the model shown in Fig. 3a of the main text are listed below (Eqs. (S45)-(S54)), with a brief description of the meaning of each one.

$$
\frac{d n_{\mathrm{P}}(t)}{d t}=\frac{v_{\mathrm{P}} n_{\mathrm{P}}(t) L}{K_{\mathrm{L}}+L}\left(1-\frac{n_{\mathrm{P}}(t)}{n_{\mathrm{P}, \max }}\right)-d n_{\mathrm{P}}(t)
$$

Eq. (S45) describes growth of the photosynthesizer population, with density $n_{\mathrm{P}}$. Photosynthesizers are located in the upper box. They grow in response to light intensity $L$ (which is a constant parameter in our model), with maximal growth rate $v_{\mathrm{P}}$ and half-saturation constant $K_{\mathrm{L}}$. We impose a population density limitation term with maximal population density $n_{\mathrm{P}, \max }$ and a death rate $d$.

$$
\frac{d n_{\mathrm{D}}(t)}{d t}=\frac{v_{\mathrm{D}} n_{\mathrm{D}}(t) C}{K_{\mathrm{C}}+C}\left(1-\frac{n_{\mathrm{D}}(t)}{n_{\mathrm{D}, \max }}\right)-d n_{\mathrm{D}}(t)
$$

Eq. (S46) describes growth of the decomposer population, with density $n_{\mathrm{D}}$. Decomposers are located in the lower box. They grow in response to organic carbon concentration $C$ (which is a constant parameter in our model), with maximal growth rate $v_{\mathrm{D}}$ and half-saturation constant $K_{\mathrm{C}}$. We impose a population density limitation term with maximal population density $n_{\mathrm{D}, \max }$ and a death rate $d$.

$$
\frac{d n_{\mathrm{or}}(t)}{d t}=v_{\mathrm{or}} n_{\mathrm{or}}(t)\left[\frac{s_{\mathrm{o}}^{\mathrm{d}}(t)}{K_{\mathrm{or}}+s_{\mathrm{o}}^{\mathrm{d}}(t)}\right]\left[\frac{a(t)}{a(t)+K_{\mathrm{ac}}}\right]\left(1-\frac{n_{\mathrm{or}}(t)}{n_{\mathrm{or}, \max }}\right)-d n_{\mathrm{or}}(t)
$$

Eq. (S47) describes growth of the microbial reducer population, with density $n_{\mathrm{or}}$. Reducers are located in the lower box. Reducer growth requires both the oxidized form of the redox chemical species, $\mathrm{s}_{\mathrm{o}}$, and acetate. We describe this using multiplicative Monod terms, with maximal growth rate $v_{\text {or }}$ and half-saturation constants $K_{\mathrm{or}}$ for $\mathrm{s}_{\mathrm{o}}$ and $K_{\mathrm{ac}}$ for acetate. The concentration of acetate is denoted as $a(t)$. The reducer population density is limited to $n_{\mathrm{or}, \mathrm{max}}$ and we impose a death rate $d$.

$$
\frac{d n_{\mathrm{ro}}(t)}{d t}=v_{\mathrm{ro}} n_{\mathrm{ro}}(t)\left[\frac{s_{\mathrm{r}}^{\mathrm{u}}(t)}{K_{\mathrm{ro}}+s_{\mathrm{r}}^{\mathrm{u}}(t)}\right]\left[\frac{o(t)}{o(t)+K_{\mathrm{ox}}}\right]\left(1-\frac{n_{\mathrm{ro}}(t)}{n_{\mathrm{ro}, \max }}\right)-d n_{\mathrm{ro}}(t)
$$

Eq. (S48) describes growth of the microbial oxidizer population, with density $n_{\text {ro }}$. Oxidizers are located in the upper box. Oxidizer growth requires both the reduced form of the redox chemical species, $\mathrm{s}_{\mathrm{r}}$, and oxygen. Again, we describe this using multiplicative Monod terms, with maximal growth rate $v_{\text {ro }}$ and half-saturation constants $K_{\text {ro }}$ for $\mathrm{s}_{\mathrm{r}}$ and $K_{\text {ox }}$ for oxygen. The concentration of oxygen is denoted as $o(t)$. The oxidizer population density is limited to $n_{\text {ro,max }}$ and we impose a death rate $d$.

$$
\frac{d o(t)}{d t}=\gamma_{\mathrm{ox}} v_{\mathrm{P}} n_{\mathrm{P}}(t)\left[\frac{L}{K_{\mathrm{L}}+L}\right]\left(1-\frac{n_{\mathrm{P}}(t)}{n_{\mathrm{P}, \max }}\right)-\gamma v_{\mathrm{ro}} n_{\mathrm{ro}}(t)\left[\frac{s_{\mathrm{r}}^{\mathrm{u}}(t)}{K_{\mathrm{ro}}+s_{\mathrm{r}}^{\mathrm{u}}(t)}\right]\left[\frac{o(t)}{o(t)+K_{\mathrm{ox}}}\right]\left(1-\frac{n_{\mathrm{ro}}(t)}{n_{\mathrm{ro}, \max }}\right)-\beta_{\mathrm{ox}} o(t)
$$


Eq. (S49) describes the dynamics of the oxygen concentration. Oxygen is assumed to be located only in the upper box. Oxygen is produced upon growth of the photosynthesizers (first term in Eq. (S49)), with yield parameter $\gamma_{\text {ox }}$ (number of micromoles of oxygen produced per bacterial division cycle). Oxygen is consumed upon growth of the oxidizer population (second term in Eq. (S49)), with yield parameter $\gamma$. We also include a term describing consumption of oxygen by other processes, not included in the model (third term in Eq. (S49)), such as growth of aerobes or abiotic oxidation reactions. The parameter $\beta_{\mathrm{ox}}$ controls the strength of this "competition" term.

$$
\frac{d a(t)}{d t}=\gamma_{\mathrm{ac}} v_{\mathrm{D}} n_{\mathrm{D}}(t)\left[\frac{C}{K_{\mathrm{C}}+C}\right]\left(1-\frac{n_{\mathrm{D}}(t)}{n_{\mathrm{D}, \max }}\right)-\gamma v_{\mathrm{or}} n_{\mathrm{or}}(t)\left[\frac{s_{\mathrm{o}}^{\mathrm{d}}(t)}{K_{\mathrm{or}}+s_{\mathrm{o}}^{\mathrm{d}}(t)}\right]\left[\frac{a(t)}{a(t)+K_{\mathrm{ac}}}\right]\left(1-\frac{n_{\mathrm{or}}(t)}{n_{\mathrm{or}, \max }}\right)-\beta_{\mathrm{ac}} a(t)
$$

Eq. (S50) describes the dynamics of the acetate concentration. Acetate is assumed to be located only in the lower box. Acetate is produced upon growth of the decomposers (first term in Eq. (S50)), with yield parameter $\gamma_{\text {ac. Acetate is }}$ consumed upon growth of the reducer population (second term in Eq. (S50)), with yield parameter $\gamma$ (here assumed to be the same as that of the reducers). We also include a "competition" term describing consumption of acetate by other processes (third term in Eq. (S50)), such as growth of methanogens. The parameter $\beta_{\text {ac }}$ controls the strength of this term.

$$
\begin{aligned}
& \frac{d s_{\mathrm{o}}^{\mathrm{u}}(t)}{d t}=\gamma v_{\mathrm{ro}} n_{\mathrm{ro}}(t)\left[\frac{s_{\mathrm{r}}^{\mathrm{u}}(t)}{K_{\mathrm{ro}}+s_{\mathrm{r}}^{\mathrm{u}}(t)}\right]\left[\frac{o(t)}{o(t)+K_{\mathrm{ox}}}\right]\left(1-\frac{n_{\mathrm{ro}}(t)}{n_{\mathrm{ro}, \max }}\right)+k s_{\mathrm{o}}^{\mathrm{d}}(t)-k s_{\mathrm{o}}^{\mathrm{u}}(t) \\
& \frac{d s_{\mathrm{o}}^{\mathrm{d}}(t)}{d t}=-\gamma v_{\mathrm{or}} n_{\mathrm{or}}(t)\left[\frac{s_{\mathrm{o}}^{\mathrm{d}}(t)}{K_{\mathrm{or}}+s_{\mathrm{o}}^{\mathrm{d}}(t)}\right]\left[\frac{a(t)}{a(t)+K_{\mathrm{ac}}}\right]\left(1-\frac{n_{\mathrm{or}}(t)}{n_{\mathrm{or}, \max }}\right)+k s_{\mathrm{o}}^{\mathrm{u}}(t)-k s_{\mathrm{o}}^{\mathrm{d}}(t) \\
& \frac{d s_{\mathrm{r}}^{\mathrm{u}}(t)}{d t}=-\gamma v_{\mathrm{ro}} n_{\mathrm{ro}}(t)\left[\frac{s_{\mathrm{r}}^{\mathrm{u}}(t)}{K_{\mathrm{ro}}+s_{\mathrm{r}}^{\mathrm{u}}(t)}\right]\left[\frac{o(t)}{o(t)+K_{\mathrm{ox}}}\right]\left(1-\frac{n_{\mathrm{ro}}(t)}{n_{\mathrm{ro}, \max }}\right)+k s_{\mathrm{r}}^{\mathrm{d}}(t)-k s_{\mathrm{r}}^{\mathrm{u}}(t) \\
& \frac{d s_{\mathrm{r}}^{\mathrm{d}}(t)}{d t}=\gamma v_{\mathrm{or}} n_{\mathrm{or}}(t)\left[\frac{s_{\mathrm{r}}^{\mathrm{d}}(t)}{K_{\mathrm{or}}+s_{\mathrm{r}}^{\mathrm{d}}(t)}\right]\left[\frac{a(t)}{a(t)+K_{\mathrm{ac}}}\right]\left(1-\frac{n_{\mathrm{or}}(t)}{n_{\mathrm{or}, \max }}\right)+k s_{\mathrm{r}}^{\mathrm{u}}(t)-k s_{\mathrm{r}}^{\mathrm{d}}(t)
\end{aligned}
$$

Eqs. (S51)-(S54) describe the dynamics of the reduced and oxidized forms of the redox chemical species, $s_{\mathrm{r}}$ and $s_{\mathrm{o}}$, in the upper and lower boxes. These equations are identical to Eqs. (S41)-(S44) for the two-population, two-box model, apart from the inclusion of multiplicative Monod terms in the reducer and oxidizer growth rates. As in the two-population, two-box model, $\mathrm{s}_{\mathrm{r}}$ and $\mathrm{s}_{\mathrm{o}}$ can be transported between the upper and lower boxes by diffusion, described by the rate parameter $k$.

Table S1 lists the parameter values used to generate the data shown in Fig. 3b of the main text. The calculations were run for different values of the light intensity $L$ (with units of $\mu$ Einstein s ${ }^{-1} \mathrm{~cm}^{-2}$ and the organic matter concentration (with units of $\mathrm{mg} \mathrm{cm}^{-3}$ ). The parameters used in these simulations were chosen to correspond approximately to the microbial sulfur cycle (see Table S1). We note that although our model does not contain anoxygenic sulfide oxidizing phototrophs (such as green sulfur bacteria), we do not expect that their inclusion would prevent redox regime shifts from occurring, and would instead move the transition point while preserving the switching behaviour. 
Table S1: Parameter values used in the model of Eqs. (S45)-(S54) to generate the data shown in Fig. 3b of the main text. These parameters are chosen to correspond approximately to the microbial sulfur cycle (with references where appropriate).

Half saturation constants and maximal growth rates are given to an order of magnitude. Growth yields are calculated assuming a bacterial mass of $10^{-12} \mathrm{~g}$. $K_{\mathrm{ox}}$ is set to $1 \mu \mathrm{M}$ as a conservative estimate, as literature values range between $1 \mu \mathrm{M}$ [5] and $20 \mu \mathrm{M}[6] . K_{\mathrm{ac}}$ is set to $1 \mu \mathrm{M}$ as a conservative estimate, to represent the fact that the electron donor could be either acetate $\left(K_{\mathrm{ac}} \sim 10 \mu \mathrm{M}\right)[7]$ or hydrogen $\left(K_{\mathrm{H}} \sim 1 \mu \mathrm{M}\right)$ [8]. Growth rates are set to $v=1 \mathrm{~h}^{-1}$ for simplicity, but we acknowledge that this would represent quite a fast growth rate for the photosynthetic and degrading populations; lower values of $v_{\mathrm{P}}$ and $v_{\mathrm{D}}$ do not affect the result. Furthermore all yields have been set to $\gamma=3 \times 10^{-8} \mu$ moles per cell for simplicity. Although in nature yield values may vary, this value is reasonable to an order of magnitude for all of the microbial populations discussed. $d$ is chosen to correspond to generic bacterial growth [9]. Redox regime shifts are not dependent on the value of $\beta_{\mathrm{ox}}$ or $\beta_{\mathrm{ac}}$, see Section S10.

\begin{tabular}{|c|c|c|}
\hline Parameter & Value & Unit \\
\hline$K_{\mathrm{L}}$ & 10 & $\mu$ Einstein $\mathrm{s}^{-1} \mathrm{~m}^{-2}[10,11]$ \\
\hline$K_{\mathrm{C}}$ & 100 & $\mathrm{mg} \mathrm{cm}^{-3}[12,13]$ \\
\hline$K_{\text {or }}$ & 1 & $\mu \mathrm{M}[14]$ \\
\hline$K_{\text {ro }}$ & 1 & $\mu \mathrm{M}[15]$ \\
\hline$K_{\text {ox }}$ & 1 & $\mu \mathrm{M}[5,6]$ \\
\hline$K_{\mathrm{ac}}$ & 1 & $\mu \mathrm{M}[7,8,16]$ \\
\hline$v_{\mathrm{P}}$ & 1 & $\mathrm{~h}^{-1}[10,17]$ \\
\hline$v_{\mathrm{D}}$ & 1 & $\mathrm{~h}^{-1}[18,19]$ \\
\hline$v_{\text {or }}$ & 1 & $\mathrm{~h}^{-1}[16]$ \\
\hline$v_{\text {ro }}$ & 1 & $\mathrm{~h}^{-1}[15]$ \\
\hline$n_{\mathrm{P}, \max }$ & $1 \times 10^{9}$ & cells per litre \\
\hline$n_{\mathrm{D}, \max }$ & $1 \times 10^{9}$ & cells per litre \\
\hline$n_{\text {or, } \max }$ & $1 \times 10^{9}$ & cells per litre [20] \\
\hline$n_{\mathrm{ro}, \max }$ & $1 \times 10^{9}$ & cells per litre [20] \\
\hline$\gamma$ & $3 \times 10^{-8}$ & $\mu$ moles per cell $[21]$ \\
\hline$\gamma_{\text {ox }}$ & $3 \times 10^{-8}$ & $\mu$ moles per cell $[22]$ \\
\hline$\gamma_{\mathrm{ac}}$ & $3 \times 10^{-8}$ & $\mu$ moles per cell $[23]$ \\
\hline$\beta_{\mathrm{ox}}$ & 0.5 & $\mathrm{~h}^{-1}$ \\
\hline$\beta_{\mathrm{ac}}$ & 0.5 & $h^{-1}$ \\
\hline$k$ & 0.1 & $\mathrm{~h}^{-1}$ \\
\hline$d$ & 0.1 & $\mathrm{~h}^{-1}$ \\
\hline
\end{tabular}

\section{Equivalent results for a biotic-abiotic model}

The equation set for an equivalent model, for a biotic-abiotic cycle (in which the reduction step is biotically-mediated but the oxidation step is abiotic), is

$$
\begin{gathered}
\frac{d n_{\mathrm{P}}(t)}{d t}=\frac{v_{\mathrm{P}} n_{\mathrm{P}}(t) L}{K_{\mathrm{L}}+L}\left(1-\frac{n_{\mathrm{P}}(t)}{n_{\mathrm{P}, \mathrm{max}}}\right)-d n_{\mathrm{P}}(t) \\
\frac{d n_{\mathrm{D}}(t)}{d t}=\frac{v_{\mathrm{D}} n_{\mathrm{D}}(t) C}{K_{\mathrm{C}}+C}\left(1-\frac{n_{\mathrm{D}}(t)}{n_{\mathrm{D}, \max }}\right)-d n_{\mathrm{D}}(t) \\
\frac{d n_{\mathrm{or}}(t)}{d t}=v_{\mathrm{or}} n_{\mathrm{or}}(t)\left[\frac{s_{\mathrm{o}}^{\mathrm{d}}(t)}{K_{\mathrm{or}}+s_{\mathrm{o}}^{\mathrm{d}}(t)}\right]\left[\frac{a(t)}{a(t)+K_{\mathrm{ac}}}\right]\left(1-\frac{n_{\mathrm{or}}(t)}{n_{\mathrm{or}, \max }}\right)-d n_{\mathrm{or}}(t) \\
\frac{d o(t)}{d t}=\gamma_{\mathrm{ox}} v_{\mathrm{P}} n_{\mathrm{P}}(t)\left[\frac{L}{K_{\mathrm{L}}+L}\right]\left(1-\frac{n_{\mathrm{P}}(t)}{n_{\mathrm{P}, \max }}\right)-v_{\mathrm{a}}\left[\frac{s_{\mathrm{r}}^{\mathrm{u}}(t)}{K_{\mathrm{a}}+s_{\mathrm{r}}^{\mathrm{u}}(t)}\right]\left[\frac{o(t)}{o(t)+K_{\mathrm{a}, \mathrm{ox}}}\right]-\beta_{\mathrm{ox}} o(t) \\
\frac{d a(t)}{d t}=\gamma_{\mathrm{ac}} v_{\mathrm{D}} n_{\mathrm{D}}(t)\left[\frac{C}{K_{\mathrm{C}}+C}\right]\left(1-\frac{n_{\mathrm{D}}(t)}{n_{\mathrm{D}, \max }}\right)-\gamma v_{\mathrm{or}} n_{\mathrm{or}}(t)\left[\frac{s_{\mathrm{o}}^{\mathrm{d}}(t)}{K_{\mathrm{or}}+s_{\mathrm{o}}^{\mathrm{d}}(t)}\right]\left[\frac{a(t)}{a(t)+K_{\mathrm{ac}}}\right]\left(1-\frac{n_{\mathrm{or}}(t)}{n_{\mathrm{or}, \max }}\right)-\beta_{\mathrm{ac}} a(t)
\end{gathered}
$$




$$
\begin{gathered}
\frac{d s_{\mathrm{o}}^{\mathrm{u}}(t)}{d t}=v_{\mathrm{a}}\left[\frac{s_{\mathrm{r}}^{\mathrm{u}}(t)}{K_{\mathrm{a}}+s_{\mathrm{r}}^{\mathrm{u}}(t)}\right]\left[\frac{o(t)}{o(t)+K_{\mathrm{a}, \mathrm{ox}}}\right]+k s_{\mathrm{o}}^{\mathrm{d}}(t)-k s_{\mathrm{o}}^{\mathrm{u}}(t) \\
\frac{d s_{\mathrm{o}}^{\mathrm{d}}(t)}{d t}=-\gamma v_{\mathrm{or}} n_{\mathrm{or}}(t)\left[\frac{s_{\mathrm{o}}^{\mathrm{d}}(t)}{K_{\mathrm{or}}+s_{\mathrm{o}}^{\mathrm{d}}(t)}\right]\left[\frac{a(t)}{a(t)+K_{\mathrm{ac}}}\right]\left(1-\frac{n_{\mathrm{or}}(t)}{n_{\mathrm{or}, \max }}\right)+k s_{\mathrm{o}}^{\mathrm{u}}(t)-k s_{\mathrm{o}}^{\mathrm{d}}(t) \\
\frac{d s_{\mathrm{r}}^{\mathrm{u}}(t)}{d t}=-v_{\mathrm{a}}\left[\frac{s_{\mathrm{r}}^{\mathrm{u}}(t)}{K_{\mathrm{a}}+s_{\mathrm{r}}^{\mathrm{u}}(t)}\right]\left[\frac{o(t)}{o(t)+K_{\mathrm{a}, \mathrm{ox}}}\right]+k s_{\mathrm{r}}^{\mathrm{d}}(t)-k s_{\mathrm{r}}^{\mathrm{u}}(t)
\end{gathered}
$$

and

$$
\frac{d s_{\mathrm{r}}^{\mathrm{d}}(t)}{d t}=\gamma v_{\mathrm{or}} n_{\mathrm{or}}(t)\left[\frac{s_{\mathrm{r}}^{\mathrm{d}}(t)}{K_{\mathrm{or}}+s_{\mathrm{r}}^{\mathrm{d}}(t)}\right]\left[\frac{a(t)}{a(t)+K_{\mathrm{ac}}}\right]\left(1-\frac{n_{\mathrm{or}}(t)}{n_{\mathrm{or}, \max }}\right)+k s_{\mathrm{r}}^{\mathrm{u}}(t)-k s_{\mathrm{r}}^{\mathrm{d}}(t) .
$$

In Eqs. (S58), (S60) and (S62), the parameter $v_{\mathrm{a}}$ is the maximal abiotic reaction rate, $K_{\mathrm{a}}$ denotes the half saturation constant with respect to $\mathrm{s}_{\mathrm{o}}$ for the abiotic oxidation reaction, and $K_{\mathrm{a}, \mathrm{ox}}$ is its half-saturation constant with respect to oxygen.

Fig. S9 shows that this biotic-abiotic cycle model can produce redox regime shifts. The steady-state global redox state of the system $\left(\left(s_{\mathrm{o}}^{\mathrm{u}}+s_{\mathrm{o}}^{\mathrm{d}}\right) / s_{\text {tot }}\right)$ is plotted as a function of the concentration $C$ of organic carbon, for the steady-state of Eqs. (S55)-(S63) as obtained by numerical solution. The parameter set used is as in Table $\mathrm{S} 1$, with $v_{\mathrm{a}}=0.2 \mu \mathrm{M}$ $\mathrm{h}^{-1}, K_{\mathrm{a}}=1 \mu \mathrm{M}$ and $K_{\mathrm{a}, \mathrm{ox}}=1 \mu \mathrm{M}, s_{\mathrm{tot}}=4 \mathrm{mM}, \beta_{\mathrm{ox}}=\beta_{\mathrm{ac}}=0.5$

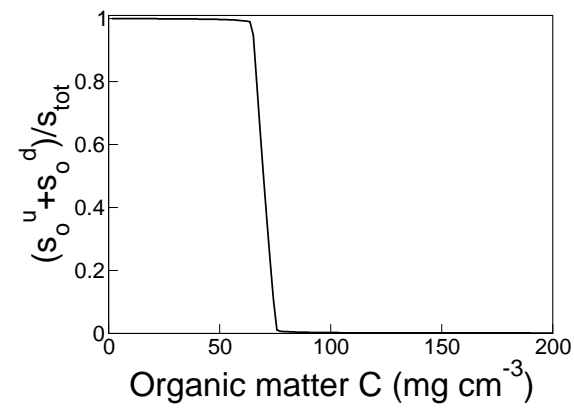

Figure S9: Redox regime shift in the biotic-abiotic model of Eqs. (S55)-(S63), obtained by numerical solution. The global redox state of the system $\left(\left(s_{\mathrm{o}}^{\mathrm{u}}+s_{\mathrm{o}}^{\mathrm{d}}\right) / s_{\text {tot }}\right)$ is plotted as a function of the concentration $C$ of organic carbon. Light intensity $\mathrm{L}$ $=20 \mu$ Einstein $\mathrm{s}^{-1} \mathrm{~m}^{-2}$. 


\section{S9. REDOX REGIME SHIFTS ARE PRESERVED WHEN INTERMEDIATE CHEMICAL OXIDATION STATES ARE INCLUDED}

In this work we have focused on models for biogeochemical cycles in which a chemical species is shuttled between two redox states. In reality, however, many biogeochemical cycles involve more than two redox states [24]; examples include the sulphur and nitrogen cycles as illustrated in Fig. 1 of the main text. Here we show that our main result, the existence of redox regime shifts, still holds if additional, intermediate redox states are included.

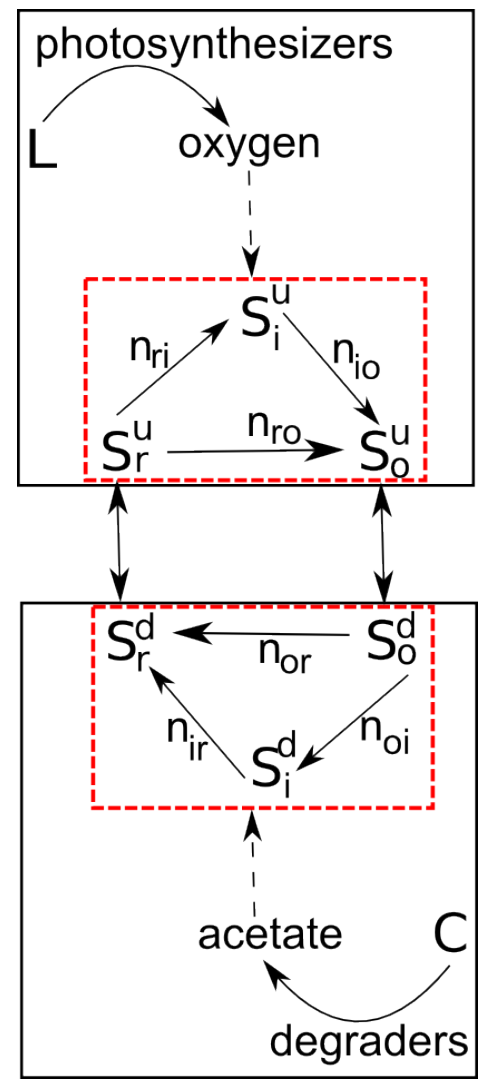

Figure S10: A microbial nutrient-cycling model with an intermediate chemical species $s_{\mathrm{i}}(t)$. An increase in oxygen availability stimulates all reactions within the upper red box, while an increase in acetate availability stimulates all reactions within the lower red box.

To investigate the effect of intermediate redox species, we extended our "4-population, 2-box" model for the fully biotic cycle to include a new chemical species, denoted $s_{i}$, whose redox state is intermediate between $s_{o}$ and $s_{r}$. We suppose that microbial populations exist that can carry out all the possible oxidative and reductive transformations: the oxidative reactions $s_{\mathrm{r}} \rightarrow s_{\mathrm{i}}, s_{\mathrm{i}} \rightarrow s_{\mathrm{O}}$ and $s_{\mathrm{r}} \rightarrow s_{\mathrm{O}}$, all of which require oxygen, and the reductive transformations $s_{\mathrm{O}} \rightarrow s_{\mathrm{i}}, s_{\mathrm{i}} \rightarrow s_{\mathrm{r}}$ and $s_{\mathrm{O}} \rightarrow s_{\mathrm{r}}$, all of which require acetate. We denote the population densities of these 6 populations $n_{\text {ri }}, n_{\text {io }}, n_{\text {ro }}, n_{\text {oi }}, n_{\text {ir }}$ and $n_{\text {or }}$, respectively. We assume that oxidative transformations only happen in the top box and reductive transformations only happen in the lower box. The two boxes are coupled by chemical diffusion.

The equations corresponding to this model are:

$$
\begin{gathered}
\frac{d n_{\mathrm{P}}(t)}{d t}=\frac{v_{\mathrm{P}} n_{\mathrm{P}}(t) L}{K_{\mathrm{L}}+L}\left(1-\frac{n_{\mathrm{P}}(t)}{n_{\mathrm{P}, \max }}\right)-d n_{\mathrm{P}}(t) \\
\frac{d n_{\mathrm{D}}(t)}{d t}=\frac{v_{\mathrm{D}} n_{\mathrm{D}}(t) C}{K_{\mathrm{C}}+C}\left(1-\frac{n_{\mathrm{D}}(t)}{n_{\mathrm{D}, \max }}\right)-d n_{\mathrm{D}}(t)
\end{gathered}
$$




$$
\begin{aligned}
& \frac{d n_{\mathrm{or}}(t)}{d t}=v_{\mathrm{or}} n_{\mathrm{or}}(t)\left[\frac{s_{\mathrm{o}}^{\mathrm{d}}(t)}{K_{\mathrm{or}}+s_{\mathrm{o}}^{\mathrm{d}}(t)}\right]\left[\frac{a(t)}{a(t)+K_{\mathrm{ac}}}\right]\left(1-\frac{n_{\mathrm{or}}(t)}{n_{\mathrm{or}, \max }}\right)-d n_{\mathrm{or}}(t) \\
& \frac{d n_{\mathrm{ri}}(t)}{d t}=v_{\mathrm{ri}} n_{\mathrm{ri}}(t)\left[\frac{s_{\mathrm{r}}^{\mathrm{u}}(t)}{K_{\mathrm{ri}}+s_{\mathrm{r}}^{\mathrm{u}}(t)}\right]\left[\frac{o(t)}{o(t)+K_{\mathrm{ox}}}\right]\left(1-\frac{n_{\mathrm{ri}}(t)}{n_{\mathrm{ri}, \max }}\right)-d n_{\mathrm{ri}}(t) \\
& \frac{d n_{\mathrm{io}}(t)}{d t}=v_{\mathrm{io}} n_{\mathrm{io}}(t)\left[\frac{s_{\mathrm{i}}^{\mathrm{u}}(t)}{\left(K_{\mathrm{io}}+s_{\mathrm{i}}^{\mathrm{u}}(t)\right)}\right]\left[\frac{o(t)}{o(t)+K_{\mathrm{ox}}}\right]\left(1-\frac{n_{\mathrm{io}}(t)}{n_{\mathrm{io}, \max }}\right)-d n_{\mathrm{io}}(t) \\
& \frac{d n_{\mathrm{oi}}(t)}{d t}=v_{\mathrm{oi}} n_{\mathrm{oi}}(t)\left[\frac{s_{\mathrm{o}}^{\mathrm{d}}(t)}{\left(K_{\mathrm{oi}}+s_{\mathrm{o}}^{\mathrm{d}}(t)\right)}\right]\left[\frac{a(t)}{a(t)+K_{\mathrm{ac}}}\right]\left(1-\frac{n_{\mathrm{oi}}(t)}{n_{\mathrm{oi}, \max }}\right)-d n_{\mathrm{oi}}(t) \\
& \frac{d n_{\mathrm{ir}}(t)}{d t}=v_{\mathrm{ir}} n_{\mathrm{ir}}(t)\left[\frac{s_{\mathrm{i}}^{\mathrm{d}}(t)}{\left(K_{\mathrm{ir}}+s_{\mathrm{i}}^{\mathrm{d}}(t)\right)}\right]\left[\frac{a(t)}{a(t)+K_{\mathrm{ac}}}\right]\left(1-\frac{n_{\mathrm{ir}}(t)}{n_{\mathrm{ir}, \max }}\right)-d n_{\mathrm{ir}}(t) \\
& \frac{d n_{\mathrm{ro}}(t)}{d t}=v_{\mathrm{ro}} n_{\mathrm{ro}}(t)\left[\frac{s_{\mathrm{r}}^{\mathrm{u}}(t)}{K_{\mathrm{ro}}+s_{\mathrm{r}}^{\mathrm{u}}(t)}\right]\left[\frac{o(t)}{o(t)+K_{\mathrm{ox}}}\right]\left(1-\frac{n_{\mathrm{ro}}(t)}{n_{\mathrm{ro}, \max }}\right)-d n_{\mathrm{ro}}(t) \\
& \frac{d o(t)}{d t}=\gamma_{\mathrm{ox}} v_{\mathrm{P}} n_{\mathrm{P}}(t)\left[\frac{L}{K_{\mathrm{L}}+L}\right]\left(1-\frac{n_{\mathrm{P}}(t)}{n_{\mathrm{P}, \max }}\right)-\beta_{\mathrm{ox}} o(t) \\
& -\gamma v_{\mathrm{ro}} n_{\mathrm{ro}}(t)\left[\frac{s_{\mathrm{r}}^{\mathrm{u}}(t)}{K_{\mathrm{ro}}+s_{\mathrm{r}}^{\mathrm{u}}(t)}\right]\left[\frac{o(t)}{o(t)+K_{\mathrm{ox}}}\right]\left(1-\frac{n_{\mathrm{ro}}(t)}{n_{\mathrm{ro}, \max }}\right) \\
& -\gamma v_{\mathrm{ri}} n_{\mathrm{ri}}(t)\left[\frac{s_{\mathrm{r}}^{\mathrm{u}}(t)}{K_{\mathrm{ri}}+s_{\mathrm{r}}^{\mathrm{u}}(t)}\right]\left[\frac{o(t)}{o(t)+K_{\mathrm{ox}}}\right]\left(1-\frac{n_{\mathrm{ri}}(t)}{n_{\mathrm{ri}, \max }}\right) \\
& -\gamma v_{\mathrm{io}} n_{\mathrm{io}}(t)\left[\frac{s_{\mathrm{i}}^{\mathrm{u}}(t)}{K_{\mathrm{io}}+s_{\mathrm{i}}^{\mathrm{u}}(t)}\right]\left[\frac{o(t)}{o(t)+K_{\mathrm{ox}}}\right]\left(1-\frac{n_{\mathrm{io}}(t)}{n_{\mathrm{io}, \max }}\right) \\
& \frac{d a(t)}{d t}=\gamma_{\mathrm{ac}} v_{\mathrm{D}} n_{\mathrm{D}}(t)\left[\frac{C}{K_{\mathrm{C}}+C}\right]\left(1-\frac{n_{\mathrm{D}}(t)}{n_{\mathrm{D}, \max }}\right)-\beta_{\mathrm{ac}} a(t) \\
& -\gamma v_{\mathrm{or}} n_{\mathrm{or}}(t)\left[\frac{s_{\mathrm{o}}^{\mathrm{d}}(t)}{K_{\mathrm{or}}+s_{\mathrm{o}}^{\mathrm{d}}(t)}\right]\left[\frac{a(t)}{a(t)+K_{\mathrm{ac}}}\right]\left(1-\frac{n_{\mathrm{or}}(t)}{n_{\mathrm{or}, \max }}\right) \\
& -\gamma v_{\mathrm{oi}} n_{\mathrm{oi}}(t)\left[\frac{s_{\mathrm{o}}^{\mathrm{d}}(t)}{K_{\mathrm{oi}}+s_{\mathrm{o}}^{\mathrm{d}}(t)}\right]\left[\frac{a(t)}{a(t)+K_{\mathrm{ac}}}\right]\left(1-\frac{n_{\mathrm{oi}}(t)}{n_{\mathrm{oi}, \max }}\right) \\
& -\gamma v_{\mathrm{ir}} n_{\mathrm{ir}}(t)\left[\frac{s_{\mathrm{i}}^{\mathrm{d}}(t)}{K_{\mathrm{ir}}+s_{\mathrm{i}}^{\mathrm{d}}(t)}\right]\left[\frac{a(t)}{a(t)+K_{\mathrm{ac}}}\right]\left(1-\frac{n_{\mathrm{ir}}(t)}{n_{\mathrm{ir}, \max }}\right) \\
& \frac{d s_{\mathrm{o}}^{\mathrm{u}}(t)}{d t}=\gamma v_{\mathrm{ro}} n_{\mathrm{ro}}(t)\left[\frac{s_{\mathrm{r}}^{\mathrm{u}}(t)}{K_{\mathrm{ro}}+s_{\mathrm{r}}^{\mathrm{u}}(t)}\right]\left[\frac{o(t)}{o(t)+K_{\mathrm{ox}}}\right]\left(1-\frac{n_{\mathrm{ro}}(t)}{n_{\mathrm{ro}, \max }}\right) \\
& +\gamma v_{\mathrm{io}} n_{\mathrm{io}}(t)\left[\frac{s_{\mathrm{i}}^{\mathrm{u}}(t)}{K_{\mathrm{io}}+s_{\mathrm{i}}^{\mathrm{u}}(t)}\right]\left[\frac{o(t)}{o(t)+K_{\mathrm{ox}}}\right]\left(1-\frac{n_{\mathrm{io}}(t)}{n_{\mathrm{io}, \max }}\right) \\
& +k s_{\mathrm{o}}^{\mathrm{d}}(t)-k s_{\mathrm{o}}^{\mathrm{u}}(t)
\end{aligned}
$$




$$
\begin{aligned}
& \frac{d s_{\mathrm{o}}^{\mathrm{d}}(t)}{d t}=-\gamma v_{\mathrm{or}} n_{\mathrm{or}}(t)\left[\frac{s_{\mathrm{o}}^{\mathrm{d}}(t)}{K_{\mathrm{ro}}+s_{\mathrm{o}}^{\mathrm{d}}(t)}\right]\left[\frac{a(t)}{a(t)+K_{\mathrm{ac}}}\right]\left(1-\frac{n_{\mathrm{or}}(t)}{n_{\mathrm{or}, \max }}\right) \\
& -\gamma v_{\mathrm{oi}} n_{\mathrm{oi}}(t)\left[\frac{s_{\mathrm{o}}^{\mathrm{d}}(t)}{K_{\mathrm{oi}}+s_{\mathrm{o}}^{\mathrm{d}}(t)}\right]\left[\frac{a(t)}{a(t)+K_{\mathrm{ac}}}\right]\left(1-\frac{n_{\mathrm{oi}}(t)}{n_{\mathrm{oi}, \max }}\right) \\
& +k s_{\mathrm{o}}^{\mathrm{u}}(t)-k s_{\mathrm{o}}^{\mathrm{d}}(t) \\
& \frac{d s_{\mathrm{r}}^{\mathrm{u}}(t)}{d t}=-\gamma v_{\mathrm{ro}} n_{\mathrm{ro}}(t)\left[\frac{s_{\mathrm{r}}^{\mathrm{u}}(t)}{K_{\mathrm{ro}}+s_{\mathrm{r}}^{\mathrm{u}}(t)}\right]\left[\frac{o(t)}{o(t)+K_{\mathrm{ox}}}\right]\left(1-\frac{n_{\mathrm{ro}}(t)}{n_{\mathrm{ro}, \max }}\right) \\
& -\gamma v_{\mathrm{ri}} n_{\mathrm{ri}}(t)\left[\frac{s_{\mathrm{r}}^{\mathrm{u}}(t)}{K_{\mathrm{ri}}+s_{\mathrm{r}}^{\mathrm{u}}(t)}\right]\left[\frac{o(t)}{o(t)+K_{\mathrm{ox}}}\right]\left(1-\frac{n_{\mathrm{ri}}(t)}{n_{\mathrm{ri}, \max }}\right) \\
& +k s_{\mathrm{r}}^{\mathrm{d}}(t)-k s_{\mathrm{r}}^{\mathrm{u}}(t) \\
& \frac{d s_{\mathrm{r}}^{\mathrm{d}}(t)}{d t}=\gamma v_{\mathrm{or}} n_{\mathrm{or}}(t)\left[\frac{s_{\mathrm{r}}^{\mathrm{d}}(t)}{K_{\mathrm{or}}+s_{\mathrm{r}}^{\mathrm{d}}(t)}\right]\left[\frac{a(t)}{a(t)+K_{\mathrm{ac}}}\right]\left(1-\frac{n_{\mathrm{or}}(t)}{n_{\mathrm{or}, \max }}\right) \\
& +\gamma v_{\mathrm{ir}} n_{\mathrm{ir}}(t)\left[\frac{s_{\mathrm{i}}^{\mathrm{d}}(t)}{K_{\mathrm{ir}}+s_{\mathrm{i}}^{\mathrm{d}}(t)}\right]\left[\frac{a(t)}{a(t)+K_{\mathrm{ac}}}\right]\left(1-\frac{n_{\mathrm{ir}}(t)}{n_{\mathrm{ir}, \max }}\right) \\
& +k s_{\mathrm{r}}^{\mathrm{u}}(t)-k s_{\mathrm{r}}^{\mathrm{d}}(t) \\
& \frac{d s_{\mathrm{i}}^{\mathrm{u}}(t)}{d t}=-\gamma v_{\mathrm{io}} n_{\mathrm{io}}(t)\left[\frac{s_{\mathrm{i}}^{\mathrm{u}}(t)}{K_{\mathrm{io}}+s_{\mathrm{i}}^{\mathrm{u}}(t)}\right]\left[\frac{o(t)}{o(t)+K_{\mathrm{ox}}}\right]\left(1-\frac{n_{\mathrm{io}}(t)}{n_{\mathrm{io}, \max }}\right) \\
& +\gamma v_{\mathrm{ri}}\left[\frac{n_{\mathrm{ri}}(t) s_{\mathrm{r}}^{\mathrm{u}}(t)}{K_{\mathrm{ri}}+s_{\mathrm{r}}^{\mathrm{u}}(t)}\right]\left[\frac{o(t)}{o(t)+K_{\mathrm{ox}}}\right]\left(1-\frac{n_{\mathrm{ri}}(t)}{n_{\mathrm{ri}, \max }}\right) \\
& +k s_{\mathrm{i}}^{\mathrm{d}}(t)-k s_{\mathrm{i}}^{\mathrm{u}}(t)
\end{aligned}
$$

and

$$
\begin{aligned}
\frac{d s_{\mathrm{i}}^{\mathrm{d}}(t)}{d t} & =-\gamma v_{\mathrm{ir}} n_{\mathrm{ir}}(t)\left[\frac{s_{\mathrm{i}}^{\mathrm{d}}(t)}{K_{\mathrm{ir}}+s_{\mathrm{i}}^{\mathrm{d}}(t)}\right]\left[\frac{a(t)}{a(t)+K_{\mathrm{ac}}}\right]\left(1-\frac{n_{\mathrm{ir}}(t)}{n_{\mathrm{ir}, \max }}\right) \\
& +\gamma v_{\mathrm{oi}} n_{\mathrm{oi}}(t)\left[\frac{s_{\mathrm{o}}^{\mathrm{d}}(t)}{K_{\mathrm{oi}}+s_{\mathrm{o}}^{\mathrm{d}}(t)}\right]\left[\frac{a(t)}{a(t)+K_{\mathrm{ac}}}\right]\left(1-\frac{n_{\mathrm{oi}}(t)}{n_{\mathrm{oi}, \max }}\right) \\
& +k s_{\mathrm{i}}^{\mathrm{u}}(t)-k s_{\mathrm{i}}^{\mathrm{d}}(t) .
\end{aligned}
$$

Here, we have defined new parameters $v_{\mathrm{oi}}, v_{\text {ir }}, v_{\text {ri }}$ and $v_{\text {io }}$, representing the maximal growth rates of the four new microbial populations, $K_{\mathrm{oi}}, K_{\mathrm{ir}}, K_{\mathrm{ri}}$ and $K_{\text {io }}$ representing their half-saturation constants with respect to their redox chemical substrate, and $n_{\text {oi,max }}, n_{\text {ir,max }}, n_{\text {ri,max }}, n_{\text {io, max }}$ representing their maximal population densities. For simplicity, we have assumed in our numerical calculations that $v_{\mathrm{oi}}=v_{\mathrm{ir}}=v_{\mathrm{ri}}=v_{\mathrm{io}}=v_{\mathrm{or}}=v_{\mathrm{ro}}$, $K_{\mathrm{oi}}=K_{\mathrm{rr}}=K_{\mathrm{ri}}=K_{\mathrm{io}}=K_{\mathrm{or}}=K_{\mathrm{ro}}$ and $n_{\mathrm{oi}, \max }=n_{\mathrm{ir}, \max }=n_{\mathrm{ri}, \max }=n_{\mathrm{io}, \max }=n_{\mathrm{or}, \max }=n_{\mathrm{ro}, \max }$, i.e. that all populations have identical growth parameters. We have also implicitly assumed in Eqs. (S64)-(S79) that all populations have equal yield parameter $\gamma$ and equal half-saturation constants with respect to oxygen or acetate $\left(K_{\mathrm{ox}}\right.$ and $\left.K_{\mathrm{ac}}\right)$. Finally we assume equal diffusion constants for all three chemical redox species.

The full parameter set used in our numerical calculations is then: $v_{\mathrm{oi}}=v_{\mathrm{ir}}=v_{\mathrm{ri}}=v_{\mathrm{io}}=v_{\mathrm{or}}=v_{\mathrm{ro}}=1 \mathrm{~h}^{-1}$, $K_{\mathrm{oi}}=K_{\mathrm{ir}}=K_{\mathrm{ri}}=K_{\mathrm{io}}=K_{\mathrm{or}}=K_{\mathrm{ro}}=1 \mu \mathrm{M}, n_{\mathrm{oi}, \max }=n_{\mathrm{ir}, \max }=n_{\mathrm{ri}, \max }=n_{\mathrm{io}, \max }=n_{\mathrm{or}, \max }=n_{\mathrm{ro}, \max }=1 \times 10^{9} \mathrm{cells}$ per litre, $k=0.1 \mathrm{~h}^{-1}, d=0.01 \mathrm{~h}^{-1}, \gamma=3 \times 10^{-8} \mu$ moles/cell, $s_{\text {tot }}=50 \mathrm{mM}, \mathrm{L}=20 \mu$ Einstein s$~^{-1} \mathrm{~m}^{-2}, K_{\mathrm{ox}}=1 \mu \mathrm{M}$, $K_{\mathrm{ac}}=1 \mu \mathrm{M}, \gamma_{\mathrm{ox}}=3 \times 10^{-8} \mu \mathrm{moles} /$ cell, $\gamma_{\mathrm{ac}}=3 \times 10^{-8} \mu \mathrm{moles} /$ cell, $\beta_{\mathrm{ox}}=\beta_{\mathrm{ox}}=0.5 h^{-1}$.

Fig. S11 shows the steady-state predictions of this model, obtained by numerical integration of Eqs. (S64)-(S79) by the Euler-forward method. This model does indeed show a regime shift as we increase the organic matter concentration (thus increasing the availability of acetate). 


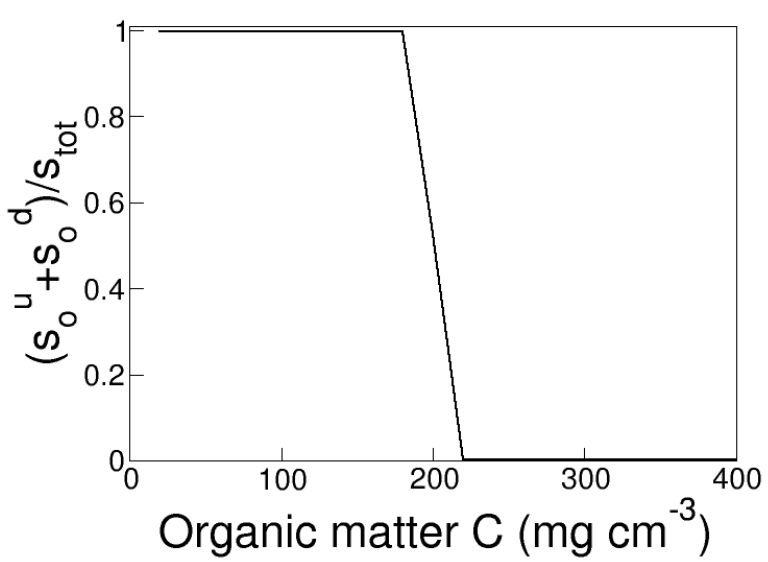

Figure S11: Redox regime shift in a model with an intermediate chemical redox state. These results were obtained by numertical solution of Eqs. (S64)-(S79) for the steady state. The global redox state of the system $\left(\left(s_{\mathrm{o}}^{\mathrm{u}}+s_{\mathrm{o}}^{\mathrm{d}}\right) / s_{\text {tot }}\right)$ is plotted as a function of the concentration $C$ of organic carbon.

\section{S10. COMPETITION FOR OXYGEN AND ACETATE}

In our four-population, two-box model, we introduced terms representing the loss of oxygen and acetate from the system (controlled by the parameters $\beta_{\mathrm{ox}}$ and $\beta_{\mathrm{ac}}$ ). These losses could be due to competition from other oxidizing or reducing microbial populations [25], abiotic processes, or diffusive loss.

Here, we show that the existence of redox regime shifts in our model is not very sensitive to the magnitude of these loss terms. Fig. S12 shows predictions of the model, Eqs. (S45)-(S54), for a range of values of $\beta_{\mathrm{ox}}=\beta_{\mathrm{ac}}$. Redox regime shifts are preserved in all cases; although the strength of the loss term affects the sharpness of the regime-shifting behaviour.

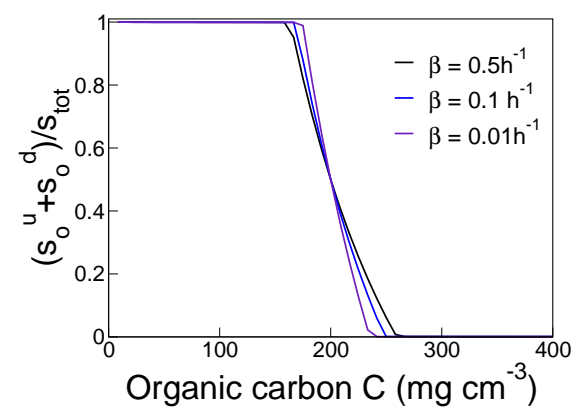

Figure S12: Redox regime shifts are not sensitive to the loss of oxygen and acetate from the system. These results were obtained by numerical solution of Eqs. (S45)-(S54) for the steady state, for various values of the loss parameter $\beta_{\mathrm{ox}}=\beta_{\mathrm{ac}}$ (here denoted $\beta)$. The global redox state of the system $\left(\left(s_{\mathrm{o}}^{\mathrm{u}}+s_{\mathrm{o}}^{\mathrm{d}}\right) / s_{\text {tot }}\right)$ is plotted as a function of the concentration $C$ of organic carbon. Parameters as shown in Table S1, with $\mathrm{L}=20 \mu$ Einstein $\mathrm{s}^{-1} \mathrm{~m}^{-2}$.

[1] Goldbeter, A. \& Koshland, D. E. Sensitivity amplification in biochemical systems. Q. Rev. Biophys 15, 555-591 (1982).

[2] Raiswell, R. \& Canfield, D. E. The iron biogeochemical cycle past present and future. Geochemical Perspectives 1 (2012).

[3] Alberghina, L., Höfer, T. \& Vanoni, M. Molecular networks and system-level properties. J. Biotechnol. 144, 224-233 (2009). 
[4] Goldbeter, A. \& Koshland, D. E. An amplified sensitivity arising from covalent modification in biological systems. Proc. Natl Acad. Sci. USA 78, 6840-6844 (1981).

[5] Klok, J. B. M. et al. A physiologically based kinetic model for bacterial sulfide oxidation. Water Research 47, 483-492 (2012).

[6] González-Sánchez, A. \& Revah, S. The effect of chemical oxidation on the biological sulfide oxidation by an alkaliphilic sulfoxidizing bacterial consortium. Enzyme. Microb. Tech. 40, 292-298 (2007).

[7] Ingvorsen, K., Zehnder, A. J. B. \& Jorgensen, B. B. Kinetics of Sulfate and Acetate Uptake by Desulfobacter postgatei. Appl. Environ. Microbiol. 47, 403-8 (1984).

[8] Kristjansson, J. K., Schonheit, P. \& Thauer, R. Different Ks values for hydrogen of methanogenic bacteria and sulfate reducing bacteria: An explanation for the apparent inhibition of methanogenesis by sulfate. Arch. Microbiol. 131, 278-282 (1982).

[9] Khatri, B., Free, A. \& Allen, R. Noise-driven oscillations in microbial population dynamics. J. Theo. Biol. 314, 120-129 (2012).

[10] Huisman, J., Pham Thi, N., Karl, D. M. \& Sommeijer, B. Reduced mixing generates oscillations and chaos in the oceanic deep chlorophyll maximum. Nature 439, 322-5 (2006).

[11] López-Urrutia, A., San Martin, E., Harris, R. P. \& Irigoien, X. Scaling the metabolic balance of the oceans. Proc. Natl. Acad. Sci. USA. 103, 8739-44 (2006).

[12] Allison, S. D., Wallenstein, M. D. \& Bradford, M. A. Soil-carbon response to warming dependent on microbial physiology. Nat. Geosci. 3, 336-340 (2010).

[13] Todd-Brown, K. E. O., Hopkins, F. M., Kivlin, S. N., Talbot, J. M. \& Allison, S. D. A framework for representing microbial decomposition in coupled climate models. Biogeochemistry 109, 19-33 (2012).

[14] Ingvorsen, K. \& Jorgensen, B. B. Kinetics of sulfate uptake by freshwater and marine species of Desulfovibrio. Arch Microbiol 139, 61-66 (1984).

[15] Canfield, D. E., Thamdrup, B. \& Kristensen, E. Aquatic Geomicrobiology. Adv. Mar. Biol. 48, $347-357$ (2005).

[16] Lovely, D. R., Dwyer, D. F. \& Klug, M. J. Kinetic Analysis of Competition Between Sulfate Reducers and Methanogens for Hydrogen in Sediments. Appl. Environ. Microbiol. 43, 1373-1379 (1982).

[17] Aiba, S. Growth kinetics of photosyntheic microorganisms. Adv. Biochem. Eng. 23, 85-156 (1982).

[18] Schou, C., Rasmussen, G., Kaltoft, M., Henrissat, B. \& Schulein, M. Stereochemistry, specificity and kinetics of the hydrolysis of reduced cellodextrins by nine cellulases. Eur. J. Biochem. 217, 947-953 (1993).

[19] Amore, A. et al. Cloning and recombinant expression of a cellulase from the cellulolytic strain Streptomyces sp G12 isolated from compost. Microbial Cell Factories 111 (2012).

[20] Foti, M. et al. Diversity, activity, and abundance of sulfate-reducing bacteria in saline and hypersaline soda lakes. Applied and Environmental Microbiology 73, 2093-2100 (2007).

[21] Jin, Q., Roden, E. E. \& Giska, J. R. Geomicrobial Kinetics: Extrapolating Laboratory Studies to Natural Environments. Geomicrobiol. J. 30, 173-185 (2013).

[22] Osborne, B. A. \& Geider, R. J. The minimum photon requirement for photosynthesis. New Phytol. 106, 631-645 (1987).

[23] Hu, Z., Wang, G. \& Yu, H. Anaerobic degradation of cellulose by rumen microorganisms at various pH values. Biochem. Eng. J. 21, 59-62 (2004).

[24] Madigan, M. T., Martinko, J. M., Dunlap, P. V. \& Clark, D. P. Brock Biology of Microorganisms (Pearson Education Inc, 2009), 12th edn.

[25] Jorgensen, B. B. Mineralization of organic matter in the sea bed - the role of sulphate reduction. Nature 296 (1982). 